\title{
GROUP-INVARIANT PERCOLATION ON GRAPHS
}

\author{
I. Benjamini, R. Lyons, Y. Peres, And O. Schramm
}

\begin{abstract}
Let $G$ be a closed group of automorphisms of a graph $X$. We relate geometric properties of $G$ and $X$, such as amenability and unimodularity, to properties of $G$-invariant percolation processes on $X$, such as the number of infinite components, the expected degree, and the topology of the components. Our fundamental tool is a new masstransport technique that has been occasionally used elsewhere and is developed further here.

Perhaps surprisingly, these investigations of group-invariant percolation produce results that are new in the Bernoulli setting. Most notably, we prove that critical Bernoulli percolation on any nonamenable Cayley graph has no infinite clusters. More generally, the same is true for any nonamenable graph with a unimodular transitive automorphism group.

We show that $G$ is amenable iff for all $\alpha<1$, there is a $G$-invariant site percolation process $\omega$ on $X$ with $\mathbf{P}[x \in \omega]>\alpha$ for all vertices $x$ and with no infinite components. When $G$ is not amenable, a threshold $\alpha<1$ appears. An inequality for the threshold in terms of the isoperimetric constant is obtained, extending an inequality of Häggström for regular trees.

If $G$ acts transitively on $X$, we show that $G$ is unimodular iff the expected degree is at least 2 in any $G$-invariant bond percolation on $X$ with all components infinite.

The investigation of dependent percolation also yields some results on automorphism groups of graphs that do not involve percolation.
\end{abstract}

\section{Introduction}

There are intimate connections between the geometry of a space or a group and the behavior of stochastic processes in it. A well-known example is

\footnotetext{
Research partially supported by the Institute for Advanced Studies, Jerusalem (Lyons), NSF grant DMS-94-04391 (Peres), and the Sam and Ayala Zacks Professorial Chair (Schramm).
} 
Kesten's theorem ([Kes1,2]) that a countable group $G$ is amenable iff some (or every) symmetric group-invariant random walk with support generating $G$ has spectral radius 1 . Here, we study the relations between a group $G$ acting on a graph $X$ and $G$-invariant percolation processes on $X$. At least as important as our particular results is the technique we use: this is a mass-transport technique that provides a good method for averaging on nonamenable graphs.

Let $G$ be a finitely generated group and let $X(G)=(V, E)$ be its right Cayley graph with respect to a (finite) set of generators. Note that left multiplication by $G$ gives a left action on $X$ by automorphisms. We call a probability measure on subsets (also called configurations) $\omega$ of $V$ a site percolation. If the distribution of $\omega$ is invariant under the left action of $G$, we call the percolation $G$-invariant. The usual case of Bernoulli percolation, in which the events $x \in \omega$ are independent and have the same probability for all vertices $x$, is, of course, an example of invariant percolation. A component of $\omega$ is a connected component for the subgraph of $X$ gotten by adding to $\omega$ all edges in $E$ whose endpoints are in $\omega$. Denote by $o$ the vertex in $X$ corresponding to, say, the identity in $G$.

The following basic result is an analogue for group-invariant percolation of Kesten's spectral radius theorem.

Theorem 1.1 (Existence of threshold). Let $X=X(G)$ be the Cayley graph of a finitely generated group $G$. Then $G$ is amenable iff for all $\alpha<1$, there is a $G$-invariant site percolation on $X$ with $\mathbf{P}[o \in \omega]>\alpha$ and with no infinite components.

The "only if" part of Theorem 1.1 follows from the Rokhlin lemma of Ornstein and Weiss ([OWe]), and the method of Adams and Lyons ([ALy]) can be used to prove the other direction. We will give another proof and a generalization in Theorem 5.1.

A quantitative form of Kesten's equivalence was provided by Cheeger $([\mathrm{C}])$ in the continuous setting; he gave a lower bound for the bottom of the spectrum of the Laplacian in terms of the isoperimetric (or "Cheeger") constant. In the discrete case, such a bound is equivalent to an upper bound on the spectral radius of the transition operator $P$, since the Laplacian is $I-P$. Cheeger's result was transferred to the discrete setting in various contexts by Dodziuk ([D]), Dodziuk and Kendall ([DKen]), Varopoulos ([V]), Ancona $([\mathrm{An}])$, Gerl ([Ge]), Biggs, Mohar, and Shawe-Taylor ([BiMoSh]), and Kaimanovich $([\mathrm{K}])$.

We have found a quantitative version of Theorem 1.1, which we now 
present. Given $K \subset V$, set $\partial_{V} K:=\{y \notin K: \exists x \in K, x \sim y\}$ and $\partial_{E} K:=\{(x, y): x \sim y, x \in K, y \notin K\}$. Define the vertex-isoperimetric constant of $X$ by

$$
\iota_{V}(X):=\inf \left\{\frac{\left|\partial_{V} K\right|}{|K|}: K \subset V \text { is finite }\right\},
$$

and let the edge-isoperimetric constant of $X$ be

$$
\iota_{E}(X):=\inf \left\{\frac{\left|\partial_{E} K\right|}{|K|}: K \subset V \text { is finite }\right\} .
$$

Thus, Følner's theorem is that a finitely generated group $G$ is amenable iff the isoperimetric constants of $X(G)$ are 0 . Let $d$ be the degree of vertices in the Cayley graph $X(G)$, i.e., the number of generators of $G$ (and their inverses) used to define the Cayley graph. Besides site percolation, we also consider bond percolation, i.e., a probability measure on subsets of $E$. (Note that every site percolation induces a bond percolation consisting of the edges joining the sites that are present.)

Theorem 1.2 (Quantitative thresholds). If $\mathbf{P}$ is a $G$-invariant site percolation on $X(G)$ and $\mathbf{P}[o \in \omega] \geqslant d /\left(d+\iota_{V}(X)\right)$, then there is an infinite component with positive probability. For $G$-invariant bond percolation, the same conclusion holds if the expected degree of each vertex is at least $d-\iota_{E}(X)$.

(See Theorems 2.12 and 2.4.)

The paper [BLySch] is largely based on the methods and results of the present paper. One of the results there is an extension of Theorem 1.2; namely, that if the weak inequality for the expected degree of bond percolation in Theorem 1.2 is replaced by a strict inequality, then with positive probability, the percolation subgraph contains a subgraph with positive isoperimetric constant.

The key tool in proving Theorem 1.2, and many other results below, is a "mass-transport" technique that we learned from Häggström ([H1]), but which was used earlier by Adams ([A]) and by van den Berg and Meester $([\mathrm{BeM}])$. It is a method that is especially useful for averaging on nonamenable graphs (though it is not restricted to nonamenable situations). Häggström used this technique in order to prove Theorem 1.2 for trees $X$; he showed that the thresholds are sharp there. Our Mass-Transport Principle is given in section 3, following reasoning of Woess ([Wo2]). Briefly, the technique is to put "mass" at the vertices, possibly depending on the percolation configuration, and then redistribute it in some fashion. A sort 
of conservation-of-mass principle then allows one to compare the mass at a vertex before and after redistribution.

For an example of invariant but not Bernoulli percolation, consider a Cayley graph $X$ and a positive integer $r$. Independently color each site red with probability $1 / 2$. Now let $\omega$ consist of those sites $x$ such that the proportion of red sites within distance $r$ of $x$ is between $40 \%$ and $60 \%$. It follows from Theorem 1.2 that if $G$ is nonamenable, then there is an easily estimable $r$ such that there is an infinite component of $\omega$ with positive probability. For example, if $d /\left(d+\iota_{V}(X)\right) \leqslant 0.95$, then it suffices to take $r$ so that the number of group elements within distance $r$ of the identity is at least 100 .

Other examples of group-invariant percolation include the connected components of plus spins in Ising models; random spanning forests of various kinds (see, e.g., [Pe], [Al], [H2], and [BLPS1]); and the random cluster model (see, e.g., [Gr]).

A graph $X$ that does not necessarily arise as a Cayley graph is called amenable if $\iota_{E}(X)=0$, or, equivalently if $X$ has bounded degree, $\iota_{V}(X)=0$. (All graphs are assumed without further comment to be countable and locally finite.) We say that a group $G \subseteq \operatorname{Aut}(X)$ is transitive or acts transitively if $|G \backslash V|=1$; we say that $G$ is quasi-transitive or acts quasitransitively if $|G \backslash V|<\infty$. (Here, the set of vertex $G$-orbits is denoted $G \backslash V$.) In these cases, one is often interested in $G$-invariant random walks on $X$. The extension of Kesten's theorem to this setting involves unimodularity and has been studied by Soardi and Woess ([SoWo]), Salvatori ([Sa]), and Saloff-Coste and Woess $([\mathrm{SWo}])$. Likewise, Theorems 1.1 and 1.2 extend to the quasi-transitive case and unimodularity plays a key role; see Theorems 4.1 and 4.4. A review of unimodularity and amenability for automorphism groups is given in section 3 .

A Bernoulli $(p)$ site [bond] percolation on a graph $X=(V, E)$ is a probability measure on subsets $\omega$ of $V$ [respectively $E]$ such that $\mathbf{P}[x \in \omega]=p$ and the events $x \in \omega$ are all independent. The parameter $p$ is sometimes called the survival parameter. The critical probability $p_{c}$ is the infimum over all $p \in[0,1]$ such that in $\operatorname{Bernoulli}(p)$ percolation, there is positive probability for the existence of an infinite connected component in $\omega$. Critical Bernoulli percolation is Bernoulli percolation with $p=p_{c}$.

The main long-standing open question in percolation is to show that critical percolation in $\mathbb{Z}^{d}$ a.s. has no infinite components for all $d \geqslant 2$. The work of Harris $([\mathrm{Har}])$ and Kesten $([\mathrm{Kes} 3])$ established the two-dimensional 
case; Hara and Slade $([\mathrm{HaSl}])$ proved it for $d \geqslant 19$. As an application of our study of group-invariant percolation, we obtain the following result (see section 7):

Theorem 1.3 (Critical percolation is finite). Let $X=(V(X), E(X))$ be a Cayley graph of a finitely generated nonamenable group, and consider either site or bond percolation on $X$. Then at the corresponding critical value $p=p_{c}$, almost surely there is no infinite cluster.

More generally, the same is true when $X$ is a graph having a transitive unimodular automorphism group.

In particular, there are no infinite components for critical percolation on hyperbolic lattices, nor on any $(k$-regular tree $) \times \mathbb{Z}$. The latter was previously known only for $k \geqslant 7$ ([Wu]). Wu's proof goes along the lines of the high-dimensional Euclidean proof and uses the triangle condition.

If one is interested only in Theorem 1.3, then certain simplifications are possible, since only Bernoulli percolation is involved. A self-contained proof of Theorem 1.3 is given in the expository note [BLPS2].

A result of independent interest that we use in the proof of Theorem 1.2 (but not in the proof of Theorem 1.3) is that no finite subset achieves the isoperimetric constant in transitive graphs:

Proposition 1.4 (Isoperimetric constants are not achieved). If $X$ is an infinite transitive graph, then for all finite $K \subset V$, we have $\left|\partial_{E} K\right| /|K|>$ $\iota_{E}(X)$ and $\left|\partial_{V} K\right| /|K|>\iota_{V}(X)$.

(See Corollaries 2.2 and 2.11.)

We also give the following characterization of amenability that involves the type of spanning subgraphs on which a group-invariant distribution can be carried. (A subgraph is spanning if it is connected and includes all vertices.)

Theorem 1.5 (Amenability, spanning trees and $p_{c}$ ). Let $G$ be a closed unimodular subgroup of $\operatorname{Aut}(X)$ that acts transitively on $X$. Then the following are equivalent:

(i) $X$ is amenable;

(ii) there is a $G$-invariant random spanning tree of $X$ with at most 2 ends a.s.;

(iii) there is a $G$-invariant random nonempty connected subgraph of $X$ with $p_{c}=1$ with positive probability.

(See Theorem 5.3.) We show by example that the theorem fails without the assumption that $G$ is unimodular. 
In section 6 , we characterize unimodularity in terms of the expected degree of vertices in infinite components. Since any connected finite graph with vertex set $V$ has average degree at least $2-2 /|V|$, one might expect that for $G$-invariant percolation on a transitive graph $X$ with all components infinite a.s., the expected degree of a vertex is at least 2 . This inequality is true when the group $G$ is unimodular, but surprisingly, whenever $G$ is not unimodular, there is a $G$-invariant percolation where the inequality fails. (See Theorem 6.1.)

In section 7 , we find a relationship between the number of ends of the components in invariant percolation and the expected degree of vertices (where "degree" refers to the degree in the percolation). Suppose that $G$ acts transitively on $X$ and is unimodular. Consider a $G$-invariant bond percolation on $X$, and let $\omega$ be its configuration. In Corollary 7.5, we show that if a.s. every component of $\omega$ is an infinite tree with finitely many ends, then the expected degree of every vertex is 2 . Towards the end of section 7 , Theorem 1.3 is proved.

In section 8 , we consider a vertex-transitive tiling $X$ of the hyperbolic plane and show that the number of infinite components of any automorphism-invariant bond percolation on $X$ is either 0,1 or $\infty$. Consequently, in any automorphism-invariant 2-coloring of the vertices of $X$, if both colors have infinite components, then a.s. at least one color must have infinitely many infinite clusters. For Bernoulli percolation on tilings of large genus, this was proved by Lalley $([\mathrm{L}])$.

The history of the study of group-invariant percolation is as follows. That the usual independence assumption in percolation theory can be profitably relaxed to group invariance was demonstrated in the remarkable proof of uniqueness of the infinite cluster, discovered by Burton and Keane ([BuKe1]). Although they considered only the case of Euclidean lattices, their proof is valid in any amenable transitive graph, provided that cubes are replaced by Følner sets; see Gandolfi, Keane, and Newman ([GKeN]). Topological consequences of invariance for percolation in $\mathbb{Z}^{2}$ were explored by Burton and Keane ([BuKe2]). The first analysis of percolation on a nonamenable graph that is not a tree was that of Bernoulli percolation on the Cartesian product of a regular tree and the integers, studied by Grimmett and Newman $([\mathrm{GrN}])$. They proved that for some $p>p_{c}$, multiple infinite clusters coexist. As a consequence of a method for studying random walks, Lyons ([Ly]) gave a threshold for Bernoulli percolation on groups of exponential growth: if $p>\lim \left|B_{n}\right|^{-1 / n}$, then there is an infinite compo- 
nent with positive probability in both Bernoulli site and bond percolation with survival parameter $p$; here $B_{n}$ is the ball of radius $n$. Benjamini and Schramm ([BSch1]) gave a threshold for Bernoulli percolation on general nonamenable graphs: if $p>1 /\left(1+\iota_{V}(X)\right)$, then there is an infinite component with positive probability in Bernoulli site percolation with parameter $p$. Likewise, if $p>1 /\left(1+\iota_{E}(X)\right)$, then there is an infinite component with positive probability in Bernoulli bond percolation with parameter $p$. As we already mentioned, Häggström $([\mathrm{H} 1])$ proved Theorem 1.2 for regular trees. His paper was the original impetus for the present work. Other studies of percolation in nonamenable settings, particularly in the hyperbolic plane, were carried out by Lalley $([\mathrm{L}])$ and by Benjamini and Schramm $([\mathrm{BSch} 2,4])$.

All three thresholds that we have mentioned (of Lyons, of Benjamini and Schramm, and of Häggström) are sharp for trees. We do not know whether the threshold for bond percolation in Theorem 1.2 is sharp for every nonamenable Cayley graph.

\section{Nonamenable Cayley Graphs}

We shall treat first the case of bond percolation on Cayley graphs, as the mass-transport method is particularly transparent there.

For an edge $e=(u, v)$, write $\check{e}:=(v, u)$. We call a function $\theta$ on $E$ antisymmetric if $\theta(u, v)=-\theta(v, u)$ for all (oriented) edges $e=(u, v)$. Set $d^{*} \theta(v):=\sum_{u \sim v} \theta(v, u)$.

The following proposition, modelled on Proposition 2.10 below from Benjamini and Schramm ([BSch3]), dualizes the inf in $\iota_{E}(X)$ not only to a sup, but to a max. It is a quantitative version of the main Theorem 3.1 of Block and Weinberger ([BlW]).

Proposition 2.1 (Flows and the edge-isoperimetric constant). For any graph $X$, we have

$$
\iota_{E}(X)=\max \left\{\alpha \geqslant 0: \exists \theta \forall e|\theta(e)| \leqslant 1 \text { and } \forall v d^{*} \theta(v)=\alpha\right\},
$$

where $\theta$ runs over the antisymmetric functions on $E$.

For the proof, we need some of the standard notions for flows in networks. A network is a directed multigraph $X=(V, E)$, a pair of distinguished vertices of $X, s_{0}$ and $s_{1}$, called the source and the sink, respectively, and a capacity function on the oriented edges $C: E \rightarrow[0, \infty]$. Write $e_{-}$and $e_{+}$for the tail and head of an edge $e$. For a function $\theta: E \rightarrow \mathbb{R}$ and $v \in V$, write $d^{*} \theta(v):=\sum_{e_{-}=v} \theta(e)-\sum_{e_{+}=v} \theta(e)$. A network flow is a function 
$\theta: E \rightarrow \mathbb{R}$ satisfying $0 \leqslant \theta(e) \leqslant C(e)$ for all $e \in E$ and $d^{*} \theta(v)=0$ for all $v \neq s_{0}, s_{1}$. The strength of the network flow is $d^{*} \theta\left(s_{0}\right)$. A cut of the network is a set $\Pi$ of oriented edges such that there is no oriented path from $s_{0}$ to $s_{1}$ in $X-\Pi$. Ford and Fulkerson's Max-Flow Min-Cut Theorem ([FFu]) says that in a finite network, the maximum strength of a network flow is equal to $\min _{\Pi} \sum_{e \in \Pi} C(e)$, where $\Pi$ ranges over all cuts of the network.

Proof of Proposition 2.1. Suppose that $\theta: E \rightarrow \mathbb{R}$ is antisymmetric and satisfies $\forall e|\theta(e)| \leqslant 1$ and $\forall v d^{*} \theta(v)=\alpha$. Let $K$ be finite. Then

$$
\alpha|K|=\sum_{v \in K} d^{*} \theta(v)=\sum_{e \in \partial_{E} K} \theta(e) \leqslant\left|\partial_{E} K\right| .
$$

This shows that $\alpha \leqslant \iota_{E}(X)$ and (for later use) that $\theta(e)=1$ for all $e \in \partial_{E} K$ if $\alpha=\iota_{E}(X)=\left|\partial_{E} K\right| /|K|$. Consequently,

$$
\iota_{E}(X) \geqslant \max \left\{\alpha \geqslant 0: \exists \theta \forall e|\theta(e)| \leqslant 1 \text { and } \forall v d^{*} \theta(v)=\alpha\right\} .
$$

Now suppose that $\alpha \leqslant \iota_{E}(X)$. For any finite $K \subset V$, define the network $X_{K}$ with vertices $K$ and two extra vertices, the source, $s_{0}$, and the sink, $s_{1}$. The edges of $X_{K}$ are as follows: For each edge in $X$ with both endpoints $x, y$ in $K$, put both oriented edges $(x, y)$ and $(y, x)$ in $X_{K}$; for each edge $(x, y) \in \partial_{E} K$, put the edge $\left(x, s_{1}\right)$ in $X_{K}$; and for each $x \in K$, put the edge $\left(s_{0}, x\right)$ in $X_{K}$. Give all edges $e$ incident to $s_{0}$ capacity $C(e):=\alpha$ and all other edges capacity $C(e):=1$.

We claim that there is a network flow from $s_{0}$ to $s_{1}$ in $X_{K}$ with strength $\alpha|K|$. Consider any cut $\Pi$ separating $s_{0}$ from $s_{1}$. Let $K^{\prime}$ be the vertices in $K$ that $\Pi$ separates from $s_{1}$. Then $\partial_{E} K^{\prime} \subseteq \Pi$ and $\left(s_{0}, v\right) \in \Pi$ for all $v \in K-K^{\prime}$. Therefore,

$$
\sum_{e \in \Pi} C(e) \geqslant\left|\partial_{E} K^{\prime}\right|+\alpha\left|K-K^{\prime}\right| \geqslant \iota(X)\left|K^{\prime}\right|+\alpha\left|K-K^{\prime}\right| \geqslant \alpha|K| .
$$

Thus, the claim follows from the Max-Flow Min-Cut Theorem. Note that the flow along $\left(s_{0}, v\right)$ is $\alpha$ for every $v \in K$.

Now let $K_{n}$ be finite sets increasing to $V$ and let $\theta_{n}$ be the corresponding flows of strength $\alpha\left|K_{n}\right|$ on $X_{K_{n}}$. There is a subsequence $\left\langle n_{i}\right\rangle$ such that for all edges $e \in E$, the limit $\theta(e):=\theta_{n_{i}}(e)$ exists; clearly, $\theta$ is antisymmetric when restricted to $E$. Also, $\forall e \in E|\theta(e)| \leqslant 1$ and $\forall v \in V d^{*} \theta(v)=\alpha$.

The following consequence appears to be new:

Corollary 2.2 (Isoperimetric constant not attained). If $X$ is an infinite transitive graph, then for all finite $K \subset V$, we have $\left|\partial_{E} K\right| /|K|>\iota_{E}(X)$.

Proof. Let $\theta$ be an antisymmetric function on $E$ with $|\theta(e)| \leqslant 1$ for all edges $e$ and $d^{*} \theta(v)=\iota_{E}(X)$ for all vertices $v$. Let $K \subset V$ be finite. Suppose for a 
contradiction that $\left|\partial_{E} K\right| /|K|=\iota_{E}(X)$. We may assume that $\theta$ is acyclic on $K$, i.e., that there is no cycle of oriented edges connecting vertices in $K$ on each of which $\theta>0$. (Otherwise, we modify $\theta$ by subtracting appropriate cycles.) The proof of Proposition 2.1 shows that for all $e \in \partial_{E} K$, we have $\theta(e)=1$; in particular, $\theta(e)>0$. Let $\left(v_{1}, v_{0}\right) \in \partial_{E} K$ and let $g$ be an automorphism of $X$ that carries $v_{0}$ to $v_{1}$. Write $v_{2}$ for the image of $v_{1}$ and $g K$ for the image of $K$. Since we also have $\left|\partial_{E}(g K)\right| /|g K|=\iota_{E}(X)$ and $\left(v_{2}, v_{1}\right) \in \partial_{E}(g K)$, it follows that $\theta\left(v_{2}, v_{1}\right)>0$. We may similarly find $v_{3}$ such that $\theta\left(v_{3}, v_{2}\right)>0$ and so on, until we arrive at some $v_{k}$ that equals some previous $v_{j}$ or is outside $K$. Both lead to a contradiction, the former contradicting the acyclicity of $\theta$, the latter contradicting the fact that on all edges leading out of $K$, we have $\theta>0$.

REMARK 2.3. It also follows from Proposition 2.1 that if $G$ covers $H$, then $\iota_{E}(G) \geqslant \iota_{E}(H)$.

For a finite subgraph $K \subset X$, set

$$
\alpha_{K}:=\frac{1}{|K|} \sum_{x \in K} \operatorname{deg}_{K}(x),
$$

where $\operatorname{deg}_{K}(x)$ refers to the degree of $x$ as a vertex in the graph $K$. Let

$$
\alpha(X):=\sup \left\{\alpha_{K}: K \subset X \text { is finite }\right\} .
$$

If $X$ is a regular graph of degree $d$, then

$$
\alpha(X)+\iota_{E}(X)=d
$$

Let $d$ be the number of generators of $G$, i.e., the degree of vertices in its Cayley graph $X$. Let $o$ denote the identity of $G$ as a vertex in $X$. Let $D(x)$ be the random degree of $x$ in the percolation configuration. Here, we consider a $G$-invariant bond percolation, so that all $D(x)$ are identically distributed; let $D$ be a random variable with this same distribution. Write $K(x)$ for the component of $x$ in the percolation configuration.

We are now ready to prove our first threshold theorem:

Theorem 2.4 (Bond-percolation threshold). If $\mathbf{P}$ is a bond percolation on a nonamenable Cayley graph $X=X(G)$ whose distribution is $G$-invariant and $\mathbf{E} D \geqslant \alpha(X)$, then there is an infinite component with positive probability. Moreover, there is positive probability for the bond $p_{c}$ of the percolation subgraph $\omega$ to satisfy $p_{c}(\omega) \leqslant \alpha(X) / \mathbf{E} D$.

Remark 2.5. The intuition is that if $\mathbf{P}$ gives a high mean degree, then it cannot be supported only on finite components with smaller average internal degree. 
Remark 2.6. Functions other than the degree can be used for thresholds, such as square degree.

REMARK 2.7. If the percolation is such that the components are guaranteed to belong to a class of subgraphs $\mathcal{C}$, then we can use the possibly better threshold $\alpha(X ; \mathcal{C}):=\sup \left\{\alpha_{K}: K \in \mathcal{C}\right.$ is finite $\}$. For example, if $\mathcal{C}$ contains only trees, then it is enough that $\mathbf{E} D \geqslant 2$.

REMARK 2.8. The proof shows the stronger result that $\mathbf{E}[D(o) \mid K(o)$ is finite] $<\alpha(X)$, provided $K(o)$ is finite with positive probability.

Proof of Theorem 2.4. We put mass $D(x)$ at each $x \in V$. All masses of vertices in infinite components are left alone, but the mass of a vertex in a finite component is redistributed equally among the vertices in that component (including itself). Thus, after redistribution, all vertices in a finite component $K$ have mass $\alpha_{K}$. Because $\mathbf{P}$ is $G$-invariant, this is a $G$ invariant mass transport, so that if $f(x, y)$ denotes the expected mass taken from $x$ and transported to $y$, then for all $g \in G$, we have $f(x, y)=f(g x, g y)$. The mass at $o$ before transport has mean $\mathbf{E} D$, whence

$$
\mathbf{E} D=\sum_{x \in X} f(o, x)
$$

On the other hand, the mass at $o$ after transport has mean

$$
\mathbf{E}\left[D(o) \mathbf{1}_{\{|K(o)|=\infty\}}+\alpha_{K(o)} \mathbf{1}_{\{|K(o)|<\infty\}}\right]=\sum_{y \in X} f(y, o) .
$$

By $G$-invariance of $f$, we have

$$
\sum_{x \in X} f(o, x)=\sum_{g \in G} f(o, g o)=\sum_{g \in G} f\left(g^{-1} o, o\right)=\sum_{y \in X} f(y, o),
$$

whence (using Corollary 2.2)

$$
\begin{aligned}
\mathbf{E} D & =\mathbf{E}\left[D(o) \mathbf{1}_{\{|K(o)|=\infty\}}+\alpha_{K(o)} \mathbf{1}_{\{|K(o)|<\infty\}}\right] \\
& <\mathbf{E}\left[d \mathbf{1}_{\{|K(o)|=\infty\}}+\alpha(X) \mathbf{1}_{\{|K(o)|<\infty\}}\right] \\
& =(d-\alpha(X)) p_{\infty}+\alpha(X),
\end{aligned}
$$

where $p_{\infty}:=\mathbf{P}[|K(o)|=\infty]$. [Actually, the strict inequality fails in the trivial case that $K(o)=X$ a.s.] Since $\alpha(X)<d$, the result follows, together with the estimate

$$
p_{\infty}>\frac{\mathbf{E} D-\alpha(X)}{d-\alpha(X)} .
$$

If $\mathbf{E} D>\alpha(X)$, then we can intersect with the configuration of an independent Bernoulli percolation with survival parameter $p^{\prime}=\alpha(X) / \mathbf{E} D$ and apply (2.2) to obtain that there is still an infinite component with 
positive probability. This means that $p_{c} \leqslant \alpha(X) / \mathbf{E} D<1$ with positive probability.

Remark 2.9. Although Bernoulli percolation at the thresholds given in [BSch1] can have the property that there are no infinite components a.s. (for example, on a tree), this is not the case with the threshold of Theorem 2.4.

The analogue of Proposition 2.1 for the vertex-isoperimetric constant is due to Benjamini and Schramm ([BSch3]) and involves the amount flowing along edges into vertices,

$$
\text { flow }_{+}(\theta, v):=\sum_{u \sim v}(\theta(u, v) \vee 0) .
$$

Proposition 2.10 (Flows and the vertex-isoperimetric constant). For any graph $X$, we have

$$
\iota_{V}(X)=\max \left\{\alpha \geqslant 0: \exists \theta \forall v \text { flow }+(\theta, v) \leqslant 1 \text { and } d^{*} \theta(v)=\alpha\right\},
$$

where $\theta$ runs over the antisymmetric functions on $E$.

Corollary 2.11 (Vertex-isoperimetric constant not attained). If $X$ is an infinite transitive graph, then for all finite $K \subset V$, we have $\left|\partial_{V} K\right| /|K|>$ $\iota_{V}(X)$.

Proof. Let $\theta$ be an antisymmetric function on $E$ with flow $(\theta, v) \leqslant 1$ and $d^{*} \theta(v)=\iota_{V}(X)$ for all vertices $v$. Let $K \subset V$ be finite. Suppose for a contradiction that $\left|\partial_{V} K\right| /|K|=\iota_{V}(X)$. It is easy to see that for all $v \in \partial_{V} K$, we have flow $+(\theta, v)=1$ and flow $+(-\theta, v)=\iota_{V}(X)+1$; in particular, there is some $e$ leading to $v$ with $\theta(e) \geqslant 1 / d$ and some $e$ leading away from $v$ with $\theta(e) \geqslant\left(\iota_{V}(X)+1\right) / d \geqslant 1 / d$, where $d$ is the degree in $X$. Since $X$ is transitive, the same is true for all $v \in V$. Therefore, we may find either a cycle or a bi-infinite path with all edges $e$ having the property that $\theta(e) \geqslant 1 / d$. We may then subtract $1 / d$ from $\theta$ along these edges, yielding another function $\theta^{\prime}$ that satisfies flow ${ }_{+}\left(\theta^{\prime}, v\right) \leqslant 1$ and $d^{*} \theta^{\prime}(v)=\iota_{V}(X)$ for all vertices $v$. But flow $\left(\theta^{\prime}, v\right)<1$ for some $v$, a contradiction.

Now we consider site percolation on $X$.

Theorem 2.12 (Site-percolation threshold). If $\mathbf{P}$ is a $G$-invariant site percolation on a nonamenable Cayley graph $X=X(G)$ with $\mathbf{P}[o \in \omega] \geqslant$ $d /\left(d+\iota_{V}(X)\right)$, then there is an infinite component with positive probability. Moreover, if $\mathbf{P}[o \in \omega]>d /\left(d+\iota_{V}(X)\right)$, then with positive probability, there is a component with $p_{c}<1$.

REMARK 2.13. If there are triangles in $X$, then the threshold $d /\left(d+\iota_{V}(X)\right)$ may be replaced by $d^{\prime} /\left(d^{\prime}+\iota_{V}(X)\right)$, where $d^{\prime}$ is the maximum number of neighbors of $o$ that are pairwise nonadjacent. 
Proof. At each $x \in \omega$, begin with mass 1. Write $n(x, y):=\mid\{z \in K(x)$ : $z \sim y\} \mid$ for the number of neighbors of $y$ in the component of $x$. The masses in infinite components are left alone, but those in a finite component are redistributed among those in the vertex boundary of that component proportionally to $n(\bullet, \bullet)$. That is, we use the $G$-invariant mass transport corresponding to the expected mass transfer from $x$ to $y$ of

$$
f(x, y):=\mathbf{E}\left[\mathbf{1}_{\{|K(x)|=\infty, y=x\}}+\frac{\mathbf{1}_{\left\{|K(x)|<\infty, y \in \partial_{V} K(x)\right\}} n(x, y)}{\left|\partial_{V} K(x)\right|}\right] .
$$

The mass at $o$ before transport has mean $\mathbf{P}[o \in \omega]$, which must equal its mean after transport, namely

$$
\begin{aligned}
\mathbf{E}\left[\mathbf{1}_{\{|K(o)|=\infty\}}+\sum_{x \sim o} \frac{\mathbf{1}_{\{o \notin \omega,|K(x)|<\infty\}}|K(x)|}{\left|\partial_{V} K(x)\right|}\right] & \\
& <\mathbf{P}[|K(o)|=\infty]+\frac{d(1-\mathbf{P}[o \in \omega])}{\iota_{V}(X)}
\end{aligned}
$$

(provided $\mathbf{P}[K(o)=X]<1)$. Therefore, we get the estimate

$$
\mathbf{P}[|K(o)|=\infty]>\mathbf{P}[o \in \omega]\left(1+\frac{d}{\iota_{V}(X)}\right)-\frac{d}{\iota_{V}(X)} .
$$

This proves the first assertion; the second follows as in the proof of Theorem 2.4.

\section{Basic Properties of Automorphism Groups}

We start by reviewing the modular function. A compact group has a unique left-invariant Radon probability measure, called Haar measure. It is also the unique right-invariant Radon probability measure. A locally compact group $G$ has a left-invariant $\sigma$-finite Radon measure $|\cdot|$; it is unique up to a multiplicative constant. For every $g \in G$, the measure $A \mapsto|A g|$ is left invariant, whence there is a positive number $m(g)$ such that $|A g|=m(g)|A|$ for all measurable $A$. The map $g \mapsto m(g)$ is a homomorphism from $G$ to the multiplicative group of the positive reals and is called the modular function of $G$. If $m(g)=1$ for every $g \in G$, then $G$ is called unimodular. In particular, this is the case if $G$ is countable, where Haar measure is counting measure.

The automorphism group $\operatorname{Aut}(X)$ of a (locally finite) graph $X$ is given the topology of pointwise convergence. If $G$ is a closed subgroup of Aut $(X)$, then $G$ is locally compact and the stabilizer

$$
S(x):=\{g \in G: g x=x\}
$$


of any vertex $x$ is compact.

Note that if $g u=y$, then $S(y)=g S(u) g^{-1}$, whence

$$
|S(y)|=\left|S(u) g^{-1}\right|=m(g)^{-1}|S(u)| \text {. }
$$

Thus, $G$ is unimodular iff for all $x$ and $y$ in the same orbit, $|S(x)|=|S(y)|$.

Unimodularity of a group of graph automorphisms is a simple and natural combinatorial property, as shown by Schlichting ([Sc]) and Trofimov ([Tr]). Namely, if $G \subset \operatorname{Aut}(X)$ is a closed group of automorphisms of a graph $X$ and $|\bullet|$ denotes cardinality (for subsets of $X$ ) as well as Haar measure (for subsets of $G$ ), then for any vertices $x, y \in X$,

$$
|S(x) y| /|S(y) x|=|S(x)| /|S(y)| ;
$$

thus, $G$ is unimodular iff for all $x$ and $y$ in the same orbit,

$$
|S(x) y|=|S(y) x| \text {. }
$$

Of course, when $G$ is countable and acting by multiplication on its Cayley graph $X$, unimodularity is trivial. We note that (3.2) is proved by showing that the number of cosets of $S(x) \cap S(y)$ in $S(x)$ is $|S(x) y|$, whence $|S(x)|=$ $|S(x) y| \cdot|S(x) \cap S(y)|$. See $[\operatorname{Tr}]$ for details.

ExAMPLE 3.1 (Nonunimodular trees). Here is an example of a transitive graph $X$ whose full automorphism group is unimodular but which has a closed group $G \subset \operatorname{Aut}(X)$ that is not unimodular: Let $X$ be the 3-regular tree. Fix an end $\xi$ of $X$ and let $G$ be the automorphisms preserving $\xi$.

For an example of a transitive graph $X$ whose full automorphism group is not unimodular, add to the above tree, for each vertex $x$, the edge between $x$ and its $\xi$-grandparent. These examples were described by Trofimov ([Tr]). For an example of a quasi-transitive tree whose full automorphism group is not unimodular, let $X$ be the 3-regular tree again and $\xi$ an end of $X$. Let the horocycles with respect to $\xi$ be $X_{n}$ in their natural order. Add a vertex to each edge joining $v$ to $w$ when $v \in X_{n}, w \in X_{n+1}$, and $n$ is even. If $X^{\prime}$ denotes the resulting tree, then $\operatorname{Aut}\left(X^{\prime}\right)$ is nonunimodular and has a finite number of orbits.

EXAMPLE 3.2 (A more interesting nonunimodular graph). Here is a significantly different example. Let $X_{1}$ be a 3-regular tree with edges oriented towards some distinguished end, and let $X_{2}$ be a 4-regular tree with edges oriented towards some distinguished end. Let $V$ be the cartesian product of the vertices of $X_{1}$ and the vertices of $X_{2}$. Join every $\left(u_{1}, u_{2}\right),\left(v_{1}, v_{2}\right) \in V$ by an edge if $\left[u_{1}, v_{1}\right]$ is an edge of $X_{1}$ and $\left[u_{2}, v_{2}\right]$ is an edge of $X_{2}$, and precisely one of these edges goes against the orientation. The resulting 
graph has infinitely many components, any two components being isomorphic. Each component is a transitive graph whose automorphism group is not unimodular.

Wolfgang Woess has informed us that this example was first discovered by Diestel and Leader for the purpose of providing a potential example of a transitive graph that is not quasi-isometric to any Cayley graph; however, this question is still open. See [Wo1].

Next, we review amenability. Let $G$ be any locally compact group and $L^{\infty}(G)$ be the Banach space of measurable essentially bounded real-valued functions on $G$ with respect to Haar measure. A linear functional on $L^{\infty}(G)$ is called a mean if it maps the constant function $\mathbf{1}$ to 1 and nonnegative functions to nonnegative numbers. If $f \in L^{\infty}(G)$ and $g \in G$, we write $L_{g} f(h):=f(g h)$. We call a mean $\mu$ invariant if $\mu\left(L_{g} f\right)=\mu(f)$ for all $f \in$ $L^{\infty}(G)$ and $g \in G$. Finally, we say that $G$ is amenable if there is an invariant mean on $L^{\infty}(G)$. A more concrete yet necessary and sufficient condition for amenability was given by Følner, namely, that for every nonempty compact $B \subset G$ and $\epsilon>0$, there is a nonempty compact set $A \subset G$ such that $|B A \triangle A| \leqslant \epsilon|A| ;$ see [P, Theorem 4.13]. In this case, one often refers informally to $A$ as a Følner set.

For automorphism groups of graphs, amenability has another interpretation:

Lemma 3.3 (Characterization of group-amenability). Let $X$ be a graph and $G$ be a closed subgroup of $\operatorname{Aut}(X)$. Then $G$ is amenable iff $X$ has a $G$-invariant mean.

Here, a mean on $\ell^{\infty}(V)$ is called $G$-invariant if every $f \in \ell^{\infty}(V)$ has the same mean as does $L_{g} f$ (defined as taking $x \mapsto f(g x)$ ).

Proof. This follows from, e.g., [ALy, Prop. 3.1]. We outline a more direct proof here. Fix $o \in V$. Suppose that $\mu$ is an invariant mean on $L^{\infty}(G)$. Given $f \in \ell^{\infty}(V)$, define $\widehat{f} \in L^{\infty}(G)$ by $\widehat{f}(h):=f(h o)$. It is straightforward to check that $\widehat{L_{g} f}=L_{g} \widehat{f}$ for all $g \in G$, whence the mean $f \mapsto \mu(\widehat{f})$ is $G$ invariant.

Conversely, suppose that $\mu$ is an invariant mean on $\ell^{\infty}(V)$. Given $f \in L^{\infty}(G)$, define $\bar{f} \in \ell^{\infty}(V)$ by $\bar{f}(x):=\int_{h o=x} f(h) d h /|S(o)|$, where $d h$ denotes Haar measure. Again, it is easy to check that $\overline{L_{g} f}=L_{g} \bar{f}$, so that the mean $f \mapsto \mu(\bar{f})$ is $G$-invariant.

Soardi and Woess ([SoWo]) established the following fundamental theorem for transitive graphs; the extension to quasi-transitive graphs is The- 
orem 1 of [Sa].

Theorem 3.4 (Characterization of graph-amenability). Let $X$ be a graph and $G$ be a closed quasi-transitive subgroup of $\operatorname{Aut}(X)$. Then $X$ is amenable iff $G$ is amenable and unimodular.

We shall give a new proof of this later (see Remarks 3.11 and 6.3) and extend it in Theorem 3.9. The original proof of Theorem 3.4 used random walks. Our proof is more direct, depending on the Mass-Transport Principle.

After this review, we now present the very useful Mass-Transport Principle, which replaces the simple (2.1). Let $\tau$ be some invariant process on $X$, such as invariant percolation, and let $F(u, v ; \tau) \in[0, \infty]$ be a function of $u, v \in V$ and $\tau$. Suppose that $F$ is invariant under the diagonal action of $G$; that is, $F(g u, g v ; g \tau)=F(u, v ; \tau)$ for all $g \in G$. We think of giving each vertex $v \in V$ some initial mass, possibly depending on $\tau$, then redistributing it so that $u$ sends $v$ the mass $F(u, v ; \tau)$. With this terminology, one hopes for "conservation" of mass, at least in expectation. Of course, the total amount of mass is usually infinite. Nevertheless, there is a sense in which mass is conserved; in the transitive unimodular setting, we have that the expected mass at a vertex before transport equals the expected mass at a vertex afterwards. More generally, mass needs to be weighted according to the Haar measure of the stabilizer as follows:

$$
\sum_{z \in G v} \mathbf{E} F(u, z ; \tau)|S(v)|=\sum_{y \in G u} \mathbf{E} F(y, v ; \tau)|S(y)|
$$

for every $u, v \in V$. This is the central formula of the mass-transport technique. Since $F$ enters only in expectation, however, it is more convenient to set $f(u, v):=\mathbf{E} F(u, v ; \tau)$. Then (3.4) follows from the following deterministic formula:

Mass-Transport Principle. If $X=(V, E)$ is any graph, $G \subseteq \operatorname{Aut}(X)$ is closed, $f: X \times X \rightarrow[0, \infty]$ is invariant under the diagonal action of $G$, and $u, v \in V$, then

$$
\sum_{z \in G v} f(u, z)|S(v)|=\sum_{y \in G u} f(y, v)|S(y)| .
$$

Proof. The following proof is due to Woess (personal communication, 1997); similar reasoning occurs, e.g., in [Wo2, pp.191-192]. If $d g$ denotes Haar measure, we have

$$
\sum_{z \in G v} f(u, z)|S(v)|=\sum_{z \in G v} f(u, z)|\{g \in G: g v=z\}|=\int_{G} f(u, g v) d g
$$




$$
\begin{aligned}
& =\int_{G} f\left(g^{-1} u, v\right) d g=\sum_{y \in G u} f(y, v)\left|\left\{g \in G: g^{-1} u=y\right\}\right| \\
& =\sum_{y \in G u} f(y, v)|\{g \in G: g y=u\}|=\sum_{y \in G u} f(y, v)|S(y)|
\end{aligned}
$$

Corollary 3.5 (Mass-transport for unimodular graphs). Let $X$ be a graph, $G \subseteq \operatorname{Aut}(X)$ be a closed unimodular quasi-transitive subgroup, and $f: X \times X \rightarrow[0, \infty]$ be invariant under the diagonal action of $G$. Choose a complete set $\left\{o_{1}, \ldots, o_{L}\right\}$ of representatives in $V$ of the orbits of $G$. Let $\mu_{i}:=\left|S\left(o_{i}\right)\right|$. Then

$$
\sum_{i=1}^{L} \mu_{i}^{-1} \sum_{z \in V} f\left(o_{i}, z\right)=\sum_{j=1}^{L} \mu_{j}^{-1} \sum_{y \in V} f\left(y, o_{j}\right) .
$$

Proof. The assumption that $G$ is unimodular means that $|S(y)|=\left|S\left(o_{i}\right)\right|$ for $y \in G o_{i}$. Thus, for each $i$ and $j$, the Mass-Transport Principle gives

i.e.,

$$
\sum_{z \in G o_{j}} f\left(o_{i}, z\right)\left|S\left(o_{j}\right)\right|=\sum_{y \in G o_{i}} f\left(y, o_{j}\right)\left|S\left(o_{i}\right)\right|
$$

$$
\mu_{i}^{-1} \sum_{z \in G o_{j}} f\left(o_{i}, z\right)=\mu_{j}^{-1} \sum_{y \in G o_{i}} f\left(y, o_{j}\right) .
$$

Adding these equations over all $i$ and $j$ gives the desired result.

In order to show why (3.5) corresponds to averaging in a natural way, we show how it corresponds to averaging on Følner sets when $X$ is amenable. Recall that $X$ is amenable if $\iota_{V}(X)=0$.

For a quasi-transitive amenable graph, the total mass leaving vertices in a Følner set is approximately the number of vertices in the set times the left-hand side of (3.5) if the frequency of vertices that lie in $G_{i}$ is $1 / \mu_{i}$. Likewise, the total mass entering vertices in a Følner set is approximately the number of vertices in the set times the right-hand side of (3.5). The following proposition makes this precise and thus shows how natural (3.5) is.

Proposition 3.6 (Density of orbits in amenable graphs). Let $X$ be an amenable graph and $G \subseteq \operatorname{Aut}(X)$ be a closed quasi-transitive subgroup. Choose a complete set $\left\{o_{1}, \ldots, o_{L}\right\}$ of representatives in $V$ of the orbits of $G$. Let $\mu_{i}:=\left|S\left(o_{i}\right)\right|$ and normalize Haar measure so that $\sum_{i} \mu_{i}^{-1}=1$. If $K_{n}$ is any sequence of finite subsets of vertices such that $\left|\partial_{V} K_{n}\right| /\left|K_{n}\right| \rightarrow 0$, then for all $i$,

$$
\lim _{n \rightarrow \infty} \frac{\left|G o_{i} \cap K_{n}\right|}{\left|K_{n}\right|}=\mu_{i}^{-1}
$$


Proof. By Theorem 3.4, $G$ is unimodular. Fix $i$ and let $r$ be the distance from $o_{1}$ to $o_{i}$. Let $n_{i}$ be the number of vertices in $G o_{i}$ at distance $r$ from $o_{1}$ and let $n_{1}$ be the number of vertices in $G o_{1}$ at distance $r$ from $o_{i}$. Begin with mass $n_{i}$ at each vertex $x \in G o_{1}$ and redistribute it equally among those vertices in $G o_{i}$ at distance $r$ from $x$. When $n$ is large, $\left|K_{n}\right|$ dominates the number of vertices at distance $r$ from $\partial_{V} K_{n}$. Hence, the total mass transported from vertices in $G o_{1} \cap K_{n}$ is asymptotically proportional to the total mass transported into vertices in $G o_{i} \cap K_{n}$; that is,

$$
\lim _{n \rightarrow \infty} \frac{n_{1}\left|G o_{i} \cap K_{n}\right|}{n_{i}\left|G o_{1} \cap K_{n}\right|}=1
$$

By the Mass-Transport Principle, we also have

$$
n_{i} \mu_{i}=n_{1} \mu_{1} \text {. }
$$

Since (3.6) and (3.7) hold for all $i$, their conjunction implies the desired result.

Analogues of Corollary 3.5 for nonunimodular groups can be proved the same way; we merely record the result for later use:

Corollary 3.7. Let $X$ be a graph, $G \subseteq \operatorname{Aut}(X)$ be a closed quasitransitive subgroup, and $f: X \times X \rightarrow[0, \infty]$ be invariant under the diagonal action of $G$. Choose a complete set $\left\{o_{1}, \ldots, o_{L}\right\}$ of representatives in $V$ of the orbits of $G$. Let $\mu_{i}:=\left|S\left(o_{i}\right)\right|$ and $a_{i} \geqslant 0$. Then

$$
\sum_{i=1}^{L} a_{i} \sum_{z \in V} f\left(o_{i}, z\right)=\sum_{i, j=1}^{L} a_{i} / \mu_{j} \sum_{y \in G o_{i}} f\left(y, o_{j}\right)|S(y)| .
$$

Given a set $K \subseteq V$, let

$$
|K|_{G}:=\sum_{x \in K}|S(x)|
$$

By (3.1), we have

$$
|g K|_{G}=m(g)^{-1}|K|_{G}
$$

Lemma 3.8. Let $G$ be a closed subgroup of $\operatorname{Aut}(X), o \in V$, and $A \subseteq G$ be closed. If $S(o) A=A$, then

$$
\left|A^{-1} o\right|_{G}=|A| \text {. }
$$

Proof. For each $x \in A^{-1} o$, let $g_{x}$ be an element of $A$ satisfying $g_{x}^{-1} o=x$. The hypothesis that $S(o) A=A$ means that $\left\{h \in A: h^{-1} o=x\right\}=S(o) g_{x}=$ $g_{x} S(x)$. In other words, $A$ is partitioned as $\left\langle g_{x} S(x): x \in A^{-1} o\right\rangle$, whence

$$
|A|=\sum_{x \in A^{-1} o}\left|g_{x} S(x)\right|=\sum_{x \in A^{-1} o}|S(x)|=\left|A^{-1} o\right|_{G} .
$$


We now give a result related to Theorem 3.4: Say that a transitive graph $X$ is $|\cdot|_{G}$-amenable if for all $\epsilon>0$, there is a finite $K \subset V$ such that $\left|\partial_{V} K\right|_{G}<\epsilon|K|_{G}$.

Theorem 3.9 (Weighted amenability). Let $G$ be a closed subgroup of $\operatorname{Aut}(X)$ that acts transitively on $X$. Then $G$ is amenable iff $X$ is $|\cdot|_{G^{-}}$ amenable.

Proof. Fix a basepoint $o \in V$. Suppose first that $G$ is amenable. Let $N$ be the set containing $o$ and its neighbors. Set

$$
B:=\left\{g \in G: g^{-1} o \in N\right\} .
$$

Then $N=B^{-1} O$ and for all $A \subseteq G$,

$$
\partial_{V}\left(A^{-1} o\right)=\left(A^{-1} N\right)-\left(A^{-1} o\right)=\left(A^{-1} B^{-1} o\right)-\left(A^{-1} o\right) .
$$

Note that $B$ is compact,

$$
S(o) B=B=B S(o),
$$

and $B A \supseteq A$. Given $\epsilon>0$, there is a compact $A \subset G$ such that

$$
|B A|-|A|=|B A-A| \leqslant \epsilon|A| \text {. }
$$

Because $B S(o)=B$, this inequality is also satisfied by $A^{\prime}=S(o) A \supseteq A$. Consequently, we assume, with no loss of generality, that $S(o) A=A$. Since $S(o) B=B$ and $S(o) A=A$, Lemma 3.8 gives

$$
|B A|-|A|=\left|A^{-1} B^{-1} o\right|_{G}-\left|A^{-1} o\right|_{G}=\left|\partial_{V}\left(A^{-1} o\right)\right| \text {. }
$$

By one more application of Lemma 3.8, we see that $X$ is $|\cdot|_{G}$-amenable by taking $K:=A^{-1} O$.

Conversely, suppose that $X$ is $|\cdot|_{G}$-amenable. Given $\epsilon>0$ and a compact set $B \subset G$ containing the identity of $G$, let $r$ be the maximum distance of any vertex in $B^{-1} o$ to $o$ and $k:=\sum_{d(x, o) \leqslant r}|S(x)| /|S(o)|$. There is a finite set $K \subset V$ such that

$$
\left|\partial_{V} K\right|_{G} \leqslant \epsilon|K|_{G} / k \text {. }
$$

Let $A:=\left\{g \in G: g^{-1} o \in K\right\}$. Since $S(o) A=A$, we have

$$
\begin{aligned}
|S(o) B A-A| & =|S(o) B A|-|A|=\left|A^{-1} B^{-1} o\right|_{G}-\left|A^{-1} o\right|_{G} \\
& =\left|A^{-1} B^{-1} o-A^{-1} o\right|_{G} .
\end{aligned}
$$

Now every vertex in $A^{-1} B^{-1} O-A^{-1} o$ lies within distance $r$ of some vertex in $\partial_{V}\left(A^{-1} o\right)=\partial_{V} K$. Therefore, (3.9), (3.8), and Lemma 3.8 give

$$
\begin{aligned}
|B A-A| & \leqslant|S(o) B A-A|=\left|A^{-1} B^{-1} o\right|_{G}-\left|A^{-1} o\right|_{G} \\
& \leqslant \sum_{x \in \partial_{V} K}|\{y: d(x, y) \leqslant r\}|_{G}
\end{aligned}
$$




$$
\begin{aligned}
& =\sum_{x \in \partial_{V} K}|\{y: d(o, y) \leqslant r\}|_{G}|S(x)| /|S(o)| \\
& =k\left|\partial_{V} K\right|_{G} \leqslant \epsilon|K|_{G}=\epsilon|A| .
\end{aligned}
$$

The following simple principle will allow us on several occasions to reduce the study of quasi-transitive graphs to transitive graphs.

Lemma 3.10 (Reducing quasi-transitive to transitive). Let $X$ be a graph and $G$ be a closed quasi-transitive subgroup of $\operatorname{Aut}(X)$. Let o be a vertex in $X$. Let $r$ be such that every vertex in $X$ is within distance $r$ of some vertex in Go. Form the graph $X^{\prime}$ from the vertices Go by joining two vertices by an edge if their distance in $X$ is at most $2 r+1$. Restriction of the elements of $G$ to $X^{\prime}$ yields a subgroup $G^{\prime} \subseteq \operatorname{Aut}\left(X^{\prime}\right)$. Then $X^{\prime}$ is connected, $G^{\prime}$ acts transitively on $X^{\prime}$, and we have the following equivalences:

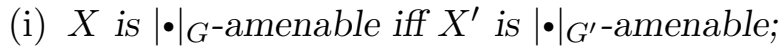

(ii) $G$ is amenable iff $G^{\prime}$ is amenable;

(iii) $G$ is unimodular iff $G^{\prime}$ is unimodular.

Proof. (i) is proved in the unimodular case by Salvatori ([Sa, Lemma 2]); the modifications needed to establish the general case are straightforward.

It is also not hard to deduce (ii) from Lemma 3.3.

To see (iii), we use (3.3). When $x, y \in X^{\prime}$, each side of (3.3) is the same whether interpreted in $G$ or in $G^{\prime}$. Hence if $G$ is unimodular, so is $G^{\prime}$. On the other hand, if $G^{\prime}$ is unimodular, then the $G$-stabilizers of the vertices in the orbit of $o$ all have the same Haar measure (by the same reasoning). Now apply (3.1) to an arbitrary element of $G$ to conclude that $G$ is unimodular.

REMARK 3.11. Together with Theorem 3.9, this immediately gives one direction of Theorem 3.4: namely, that if $G$ is amenable and unimodular, then $X$ is amenable.

The following will be useful in proving Theorem 5.1.

Lemma 3.12. Let $G$ be a closed subgroup of $\operatorname{Aut}(X)$ that acts transitively on $X$. Let $K \subset V$ be finite. For each $x \in V$, let $g_{x}$ be a random element of $G$ that takes $o$ to $x$ chosen according to normalized Haar measure. Fix some $y \in V$. Then

$$
\mathbf{E}\left|\left\{x \in V: y \in g_{x} K\right\}\right|=|K|_{G}|S(o)|^{-1} .
$$

Proof. By the Mass-Transport Principle, we have

$$
\mathbf{E}\left|\left\{x \in V: y \in g_{x} K\right\}\right| \cdot|S(o)|=\sum_{x \in V} \mathbf{P}\left[y \in g_{x} K\right]|S(o)|
$$




$$
=\sum_{z \in V} \mathbf{P}\left[z \in g_{o} K\right]|S(z)|=\mathbf{E}\left|g_{o} K\right|_{G} .
$$

By (3.1), $\left|g_{o} K\right|_{G}=m\left(g_{o}\right)^{-1}|K|_{G}=|K|_{G}$.

\section{Thresholds for Quasi-Transitive Graphs}

We now extend the threshold for percolation on Cayley graphs to quasitransitive graphs. However, in the quasi-transitive case the thresholds are not as natural as in the transitive case and there is room for improvement. For readability, we first treat the case of unimodular automorphism groups.

Theorem 4.1. Let $X$ be a graph, $G \subseteq \operatorname{Aut}(X)$ be a closed unimodular quasi-transitive subgroup, and let $o_{1}, \ldots, o_{L}$ be a complete set of representatives in $V$ of the orbits of $G$. Let $\mu_{i}:=\left|S\left(o_{i}\right)\right|$. Let $\mathbf{P}$ be a $G$-invariant bond percolation on $X$. Let $D_{i}$ denote the random degree of $o_{i}$ in the percolation subgraph and let $d_{i}$ denote the degree of $o_{i}$ in $X$. Write $p_{\infty, i}$ for the probability that $o_{i}$ is in an infinite component. Then

$$
\sum_{i=1}^{L} \mu_{i}^{-1}\left[d_{i}-\alpha(X)\right] p_{\infty, i} \geqslant \sum_{j=1}^{L} \mu_{j}^{-1}\left[\mathbf{E} D_{j}-\alpha(X)\right] .
$$

In particular, if the right-hand side of (4.1) is positive, then there is an infinite component with positive probability.

REMARK 4.2. In case $G$ acts transitively, then the $\geqslant$ in (4.1) can be replaced by $>$ provided, of course, that the percolation subgraph is not the whole graph with probability 1.

REMARK 4.3 (Threshold for forest percolation). For later use, we record Theorem 4.1 for transitive groups in the version where all components of the percolation configuration are trees (as in Remark 2.7): Let $G$ be a closed unimodular transitive subgroup of $\operatorname{Aut}(X)$. Let $\mathbf{P}$ be a $G$-invariant bond percolation on $X$ all of whose components are trees a.s. If $\mathbf{E} D \geqslant 2$, then there is an infinite component with positive probability.

Proof of Theorem 4.1. We put mass $D(x)$ at each $x \in V$. All masses of vertices in infinite components are left alone, but those in a finite component are redistributed equally among the vertices in that component. Since $\mathbf{P}$ is $G$-invariant, so is this mass transport. Formally, we use the function

$$
f(x, y):=\mathbf{E}\left[\mathbf{1}_{\{|K(x)|=\infty, y=x\}} D(x)+\mathbf{1}_{\{|K(x)|<\infty, y \in K(x)\}} \frac{D(x)}{|K(x)|}\right] .
$$


The mass at $x$ before transportation has mean $\mathbf{E} D(x)$, whence

$$
\sum_{i=1}^{L} \mu_{i}^{-1} \sum_{z \in V} f\left(o_{i}, z\right)=\sum_{i=1}^{L} \mu_{i}^{-1} \mathbf{E} D_{i}
$$

On the other hand, the mass at $z$ after transportation has mean

whence

$$
\mathbf{E}\left[D(z) \mathbf{1}_{\{|K(z)|=\infty\}}+\alpha_{K(z)} \mathbf{1}_{\{|K(z)|<\infty\}}\right],
$$

$$
\begin{aligned}
\sum_{i=1}^{L} \mu_{i}^{-1} \sum_{y \in V} f\left(y, o_{i}\right) & =\sum_{i=1}^{L} \mu_{i}^{-1} \mathbf{E}\left[D_{i} \mathbf{1}_{\left\{\left|K\left(o_{i}\right)\right|=\infty\right\}}+\alpha_{K\left(o_{i}\right)} \mathbf{1}_{\left\{\left|K\left(o_{i}\right)\right|<\infty\right\}}\right] \\
& \leqslant \sum_{i=1}^{L} \mu_{i}^{-1}\left[d_{i} p_{\infty, i}+\alpha(X)\left(1-p_{\infty, i}\right)\right] .
\end{aligned}
$$

Corollary 3.5 and a little algebra complete the proof.

An analogue of Theorem 4.1 in the (possibly) nonunimodular case is as follows: For a finite subgraph $K \subset X$, set

and

$$
\alpha_{K}^{G}:=\frac{1}{|K|_{G}} \sum_{x \in K} \operatorname{deg}_{K}(x)|S(x)|
$$

$$
\alpha^{G}(X):=\sup \left\{\alpha_{K}^{G}: K \subset X \text { is finite }\right\} .
$$

If $X$ is a regular graph of degree $d$, then

$$
\alpha^{G}(X)+\iota_{E}^{G}(X)=d
$$

where

$$
\iota_{E}^{G}(X):=\inf \left\{\frac{\sum_{(x, y) \in \partial_{E} K}|S(x)|}{|K|_{G}}: K \subset V \text { is finite }\right\} .
$$

Define the $G$-degree of a vertex $x \in X$ as $\sum_{y \sim x}|S(y)| /|S(x)|$.

Theorem 4.4. Let $X$ be a graph, $G \subseteq \operatorname{Aut}(X)$ be a closed quasi-transitive subgroup, and let $o_{1}, \ldots, o_{L}$ be a complete set of representatives in $V$ of the orbits of $G$. Let $\mathbf{P}$ be a $G$-invariant bond percolation on $X$. Let $D_{i}^{G}$ denote the random $G$-degree of $o_{i}$ in the percolation subgraph and let $d_{i}^{G}$ denote the $G$-degree of $o_{i}$ in $X$. Write $p_{\infty, i}$ for the probability that $o_{i}$ is in an infinite component. Then

$$
\sum_{i=1}^{L}\left[d_{i}^{G}-\alpha^{G}(X)\right] p_{\infty, i} \geqslant \sum_{j=1}^{L}\left[\mathbf{E} D_{j}^{G}-\alpha^{G}(X)\right] .
$$

In particular, if the right-hand side of (4.1) is positive, then there is an infinite component with positive probability. 
REMARK 4.5. Even in the unimodular case, Theorem 4.4 gives a sometimes different result than Theorem 4.1.

Proof. We merely indicate the changes necessary to the proof of Theorem 4.1. Given the percolation configuration $\omega$, put mass $D^{G}(x)$ at each $x \in V$, where $D^{G}(x)$ is the $G$-degree of $x$ in $\omega$. Redistribute the masses in finite components $G$-equally, i.e., proportionally to the weights $|S(y)|$ (for $y$ in the component). This defines $f(x, y)$; use Corollary 3.7 with $a_{i}=1$ for all $i$.

\section{Qualitative Characterizations of Amenability}

Besides the many characterizations of amenable groups in general, we have the following characterization for automorphism groups:

Theorem 5.1 (Amenability and finite percolation). Let $X$ be a graph and $G$ be a closed subgroup of $\operatorname{Aut}(X)$. Then $G$ is amenable iff for all $\alpha<1$, there is a $G$-invariant site percolation on $X$ with $\mathbf{P}[x \in \omega]>\alpha$ for all $x$ and with no infinite components.

REMARK 5.2. The analogous result for bond percolation is proved similarly.

Proof. Suppose that for all $n=1,2, \ldots$, there is some $G$-invariant site percolation with configuration $\omega_{n}$ on $X$ with $\mathbf{P}\left[o \in \omega_{n}\right] \geqslant 1-\frac{1}{n}$, but with no infinite components. Then we can define a $G$-invariant mean on $X$ by mapping $f \in \ell^{\infty}(V) \mapsto \operatorname{LIM}_{n \rightarrow \infty} \mathbf{E}_{n}\left[\sum_{x \in K(o)} f(x) /|K(o)|\right]$, where LIM is a Banach limit, $o$ is any fixed vertex in $X$, and $\mathbf{E}_{n}$ denotes expectation with respect to $\omega_{n}$. Since $X$ has a $G$-invariant mean, $G$ is itself amenable by Lemma 3.3.

Now we prove the converse. Suppose that $G$ is amenable. We consider first the case that $G$ acts transitively on $X$.

Fix a finite set $K \subset V$ and consider the following percolation. For each $x \in V$, choose a random $g_{x} \in G$ that takes $o$ to $x$ and let $Z_{x}$ be a random bit that equals 1 with probability $|S(o)| /|K|_{G}$. Choose all $g_{x}$ and $Z_{x}$ independently. Remove the sites

$$
\bigcup_{x \in X, Z_{x}=1} \partial_{V}\left(g_{x} K\right)
$$

that is, consider the percolation subgraph

$$
\omega:=\omega_{K}:=V-\bigcup\left\{\partial_{V}\left(g_{x} K\right): Z_{x}=1\right\} .
$$

Then the distribution of $\omega$ is a $G$-invariant percolation on $X$. 
We claim that

and

$$
\mathbf{P}[o \notin \omega] \leqslant\left|\partial_{V} K\right|_{G} /|K|_{G}
$$

$$
P[|K(o)|<\infty] \geqslant 1-1 / e .
$$

To prove (5.1), note that the probability that $o \notin \omega$ is at most the expected number of $x$ such that $Z_{x}=1$ and $o \in \partial_{V}\left(g_{x} K\right)$. Lemma 3.12 shows that this expectation is exactly the right-hand side of (5.1). To prove (5.2), use the independence to calculate that

$$
\begin{aligned}
\mathbf{P}[|K(o)|<\infty] & \geqslant \mathbf{P}\left[\exists x o \in g_{x} K \text { and } Z_{x}=1\right] \\
& =1-\mathbf{P}\left[\forall x \neg\left(o \in g_{x} K \text { and } Z_{x}=1\right)\right] \\
& =1-\prod_{x \in V}\left(1-\mathbf{P}\left[o \in g_{x} K\right] \mathbf{P}\left[Z_{x}=1\right]\right) \\
& =1-\prod_{x \in V}\left(1-\mathbf{P}\left[o \in g_{x} K\right]|S(o)| /|K|_{G}\right) \\
& \geqslant 1-\exp \left\{-\sum_{x \in V} \mathbf{P}\left[o \in g_{x} K\right]|S(o)| /|K|_{G}\right\} \\
& =1-\exp \left\{-\mathbf{E}\left|\left\{x \in V: o \in g_{x} K\right\}\right||S(o)| /|K|_{G}\right\} \\
& =1-1 / e
\end{aligned}
$$

by Lemma 3.12 again.

Now, since $X$ is $|\cdot|_{G}$-amenable by Theorem 3.9 , there is a sequence $K_{n}$ of finite sets of vertices with $\sum_{n}\left|\partial_{V} K_{n}\right|_{G} /\left|K_{n}\right|_{G}<1-\alpha$. For each $n$, let $\omega_{n}$ be the random subgraph from the percolation just described based on the set $K_{n}$. Choose $\omega_{n}$ to be independent and consider the percolation with configuration $\omega:=\cap \omega_{n}$. By (5.1), we have $\mathbf{P}[o \in \omega]>\alpha$ and by (5.2), we have $\mathbf{P}[|K(o)|<\infty]=1$.

This finishes the transitive case. Next, assume that $G$ is quasi-transitive. We reduce to the transitive case as follows. Let $o$ be a vertex in $X$ and form the graph $X^{\prime}$ as in Lemma 3.10. Given a $G^{\prime}$-invariant site percolation with configuration $\omega^{\prime}$ on $X^{\prime}$ that has only finite components and with $\mathbf{P}\left[o \in \omega^{\prime}\right]$ very close to 1 , form the $G$-invariant percolation with configuration $\omega$ on $X$ by keeping those $x$ such that all vertices in $X^{\prime}$ within distance $r$ of $x$ are in $\omega^{\prime}$. Trivially, $\mathbf{P}[x \in \omega]$ is very close to 1 for all $x$. If there were an infinite component in $\omega$, say, a path $\left\langle x_{1}, x_{2}, \ldots\right\rangle$, then we would be able to find an infinite component $\left\{y_{1}, y_{2}, \ldots\right\}$ in $\omega^{\prime}$ by taking $y_{i}$ to be any vertex in Go within distance $r$ of $x_{i}$.

Finally, consider the general case. Fix $o \in V$ and $0<\alpha<1$. Choose an integer $R$ at random in the range $1 /(1-\alpha)<R<2 /(1-\alpha)$. Remove 
every vertex whose distance from $G o$ is a multiple of $R$. This breaks $X$ into a disconnected union of quasi-transitive graphs. On each, we may find an invariant percolation with marginal $>\alpha$ and only finite components. Putting these together does the job.

For unimodular groups, the next theorem gives another characterization of amenability.

Theorem 5.3 (Amenability, spanning trees and $p_{c}$ ). Let $G$ be a closed subgroup of $\operatorname{Aut}(X)$ that acts transitively on $X$. Then each of the following conditions implies the next one:

(i) $X$ is amenable;

(ii) there is a $G$-invariant random spanning tree of $X$ with at most 2 ends a.s.;

(iii) there is a $G$-invariant random nonempty connected subgraph $\omega$ of $X$ that satisfies $p_{c}(\omega)=1$ with positive probability;

(iv) $G$ is amenable.

If $G$ is assumed to be unimodular, then all four conditions are equivalent.

In part (iii), to say that $\omega$ is connected means that $\omega \cap V$ is connected by edges from $\omega \cap E$.

Proof. Assume (i). Then $G$ is amenable by Theorem 3.4. From the proof of Theorem 5.1, we obtain a sequence of invariant site percolations with configurations $\tilde{\omega}_{1} \subset \tilde{\omega}_{2} \subset \cdots$ with each of them having no infinite components and with the marginals tending to 1 : namely, let $\hat{\omega}_{n}:=\bigcap_{k \geqslant n} \omega_{k}$, with $\omega_{k}$ as in the proof of Theorem 5.1. Let $\tilde{\omega}_{n}$ be the set of edges of $G$ that do not have an endpoint in $\hat{\omega}_{n}$. An invariant random spanning tree is constructed as follows. For each finite component of $\tilde{\omega}_{1}$, take a uniformly chosen spanning tree and let $T_{1}$ be their union (a forest). The finite components of $\tilde{\omega}_{1}$ partition the finite components of $\tilde{\omega}_{2}$ and thus induce a graph structure on the components of $\tilde{\omega}_{2}$. Hence a uniformly chosen spanning tree on this induced graph structure gives a spanning tree for the components of $\tilde{\omega}_{2}$; let $T_{2}$ be their union. Continue inductively and let $T:=\bigcup T_{n}$ be the union of these forests. Clearly the distribution of $T$ is $G$-invariant. We claim that, in fact, $T$ is a tree a.s. Since the marginals of $\tilde{\omega}_{n}$ tend to 1 , the probability that any two given vertices lie in the same component of $\tilde{\omega}_{n}$ also tends to 1 . Thus, with probability arbitrarily close to 1 , they are in the same component of $T$. Thus, $T$ is a tree a.s. The fact that $T$ has at most 2 ends is true for any invariant spanning tree in $X$ by the argument in [BuKe1]. Thus, we have (ii). 
The implication (ii) implies (iii) is trivial, so assume (iii). Let $\omega$ be a $G$-invariant random connected subgraph of $X$ with $p_{c}(\omega)=1$. To deduce (iv), we use the Adams-Lyons method. First, let $o_{\omega}$ be a random vertex in $\omega$. For example, one may choose $o_{\omega}$ uniformly among the closest vertices in $\omega$ to some fixed vertex $o$. Define a mean $\mu$ on $X$ by mapping

$$
f \in \ell^{\infty}(V) \mapsto \mu(f):=\operatorname{LIM}_{n \rightarrow \infty} \mathbf{E} \sum_{x \in K_{n}\left(o_{\omega}\right)} f(x) /\left|K_{n}\left(o_{\omega}\right)\right|,
$$

where LIM is a Banach limit and $K_{n}\left(o_{\omega}\right)$ denotes the cluster of $o_{\omega}$ in a Bernoulli bond percolation on $\omega$ with survival parameter $1-1 / n$. Since $\omega$ is connected, the choice of $o_{\omega}$ does not affect the value of $\mu(f)$. Thus, $\mu$ is $G$-invariant. Since $X$ has a $G$-invariant mean, $G$ is itself amenable by Lemma 3.3, proving (iv).

If $G$ is unimodular, then (iv) $\Rightarrow$ (i) by Theorem 3.4.

Note that if $X$ is a 3-regular tree and $G$ is the group of automorphisms fixing a given end $\xi$, then $G$ is amenable, but obviously the only spanning tree of $X$ is $X$ itself. Thus (iv) does not imply (ii) without assuming unimodularity.

The same example shows that (iv) does not imply (iii). In order to see this, let $\omega$ be any stationary connected nonempty subgraph of $X$. Suppose that with positive probability, $\omega$ does not contain a path to $\xi$. Conditioned on this event, there is a unique vertex of $\omega$ which is closest to $\xi$. This allows a $G$-invariant choice of a vertex in $X$, which is impossible. Consequently, $\omega$ a.s. contains a path leading to $\xi$. Fix any vertex $v \in V$, and let $v_{n}$ be the vertex on the path from $v$ to $\xi$ at distance $n$ from $v$. Then the probability that $v_{n} \in \omega$ tends to 1 as $n \rightarrow \infty$. Since the probability of a vertex to be in $\omega$ is independent of the vertex, this means that $v \in \omega$ a.s.; that is, $\omega=X$ a.s.

We now present an example showing that (ii) does not imply (i). Let $X$ and $G$ be as above. On $\mathbb{Z}^{2}$ consider the following percolation. Let $\left\langle x_{v}\right\rangle$ be i.i.d. random variables indexed by the vertices of $\mathbb{Z}^{2}$, with $x_{v}=(0,1)$ or $x_{v}=(1,0)$, each with probability $1 / 2$. Let $\omega$ be the bond percolation on $\mathbb{Z}^{2}$ defined by

$$
\omega:=\left\{\left[v, v+x_{v}\right]: v \in \mathbb{Z}^{2}\right\} .
$$

This percolation on $\mathbb{Z}^{2}$ is essentially the same as one constructed by Tóth and Werner ([TWer]), and can be interpreted as the wired spanning forest for a Markov chain on $\mathbb{Z}^{2}$. It is immediate to verify that $\omega$ is a.s. connected. Let $\omega^{\dagger}$ be the set of edges of the planar dual of $\mathbb{Z}^{2}$ that do not cross an edge in $\omega$. Then the distribution of $\omega^{\dagger}$ is isomorphic to the distribution of $\omega$. By 
a simple duality argument, the fact that $\omega^{\dagger}$ is connected a.s. implies that $\omega$ has a single end a.s. (compare [BLPS1, Section 12]). Let $f_{1}: V(X) \rightarrow \mathbb{Z}$ be a map such that geodesic rays in $X$ tending to $\xi$ are mapped by $f_{1}$ to geodesic rays in $\mathbb{Z}$ tending to $+\infty$ (and $f_{1}$ takes neighbors in $X$ to neighbors in $\mathbb{Z})$. Let $f: V(X \times \mathbb{Z}) \rightarrow \mathbb{Z}^{2}$ be defined by $f(x, n):=\left(f_{1}(x), n\right)$. Finally, let $f^{*} \omega$ be the pullback of $\omega$ by $f$; that is, an edge is in $f^{*} \omega$ iff the images of its endpoints are connected by an edge in $\omega$. Then it is not hard to verify that $f^{*} \omega$ is almost surely a tree with one end whose distribution is invariant under $G \times \mathbb{Z}$, where $\mathbb{Z}$ acts on itself by translations. Because $X \times \mathbb{Z}$ is not amenable, we find that (ii) does not imply (i).

Question. Does the implication (iii) $\Rightarrow$ (ii) hold?

Remark 5.4. Recently, R. Pemantle and Y. Peres ([PePer $]$ ) have shown that if $X$ and $Y$ are quasi-transitive infinite graphs and at least one of them is nonamenable, then there is no automorphism-invariant random spanning tree in the Cartesian product $X \times Y$.

Corollary 5.5 (Uniqueness only above $p_{c}$ ). If $X$ is a transitive graph with a nonamenable automorphism group and Bernoulli (site or bond) percolation with survival parameter $p$ produces a unique infinite component a.s., then $p>p_{c}(X)$.

Proof. Suppose that $p=p_{c}(X)$ and that there is a unique infinite component a.s. Then the infinite component $\omega$ has $p_{c}(\omega)=1$ a.s. Hence $G$ is amenable.

The argument in [BuKe1] that we used in the proof of Theorem $5.3 \mathrm{im}$ plies that any invariant percolation on an amenable transitive graph yields only components with at most 2 ends. The converse is true as well, namely, on any nonamenable transitive graph, there is an invariant percolation that a.s. yields a component with at least 3 ends. This is proved in [BLPS1]. The proof of this characterization of amenability is related to another characterization that appears in that paper: Let $X$ be a transitive graph. If $T$ denotes the wired uniform spanning forest and $\omega_{p}$ is the configuration of independent Bernoulli bond percolation with survival parameter $p$, then $X$ is amenable iff for every $p>0$, the union $T \cup \omega_{p}$ is connected a.s.

\section{Characterization of Unimodularity}

Theorem 6.1 (Modulus and expected degree). Let $G$ be a closed subgroup of $\operatorname{Aut}(X)$ that acts transitively on $X$. Let $m$ be the minimum of 
$|S(x)| /|S(y)|$ for $x, y$ neighbors. Then for any $G$-invariant percolation that yields infinite components with positive probability, the expected degree of $x$ given that $x$ is in an infinite component is at least $1+m$. This is sharp for all pairs $(G, X)$ in the sense that there is a $G$-invariant bond percolation on $X$ with every vertex belonging to an infinite component and having expected degree $1+m$.

Proof. Begin with mass 1 at each vertex. Transport the mass at $x$ to $y$ if

(1) $x$ and $y$ are adjacent;

(2) $x$ and $y$ are both in an infinite component; and

(3) if the edge $(x, y)$ is removed, then $x$ is isolated to a finite component.

Note that for each $x$ in an infinite component, there is at most one vertex to which the mass at $x$ might be transported. Also, there are at most $D(x)-1$ vertices that might transport their mass to $x$. Define $\theta(x, y)$ to be the mass transported from $x$ to $y$ and, as usual, $f(x, y):=\mathbf{E}[\theta(x, y)]$.

Fix $x$. By the Mass-Transport Principle, it follows that $\mathbf{E}[\Delta]=0$, where

$$
\Delta:=\sum_{y \in V} \theta(x, y)-\sum_{z \in V} \theta(z, x)|S(z)| /|S(x)| .
$$

Now $\Delta=1-1=0$ if $K(x)$ is finite. If $K(x)$ is infinite, then consider three cases: (1) If $D(x)=1$, then $\Delta=1$. (2) If $D(x) \geqslant 2$ and for some $y \neq x$, we have $\theta(x, y)=1$, then $\sum_{y \in V} \theta(x, y)=1$ and $\sum_{z \in V} \theta(z, x)|S(z)| /|S(x)| \leqslant$ $(D(x)-1) / m$, whence

$$
\Delta \geqslant 1-(D(x)-1) / m
$$

(3) If $D(x) \geqslant 2$ and for all $y \neq x$, we have $\theta(x, y)=0$, then $\sum_{z \in V} \theta(z, x)|S(z)| /|S(x)| \leqslant(D(x)-2) / m$; since $m \leqslant 1$, again (6.1) holds. Thus, in all three cases, (6.1) holds. Taking expectation, we obtain that

$$
0=\mathbf{E}[\Delta]=\mathbf{E}[\Delta ;|K(x)|=\infty] \geqslant \mathbf{E}[1-(D(x)-1) / m ;|K(x)|=\infty],
$$

which is the same as $\mathbf{E}[D(x)|| K(x) \mid=\infty] \geqslant 1+m$.

To show that this bound is sharp in case $G$ is not unimodular, i.e., $m<1$, consider the following percolation. For each vertex $x$, let

$$
L(x):=\{y \sim x:|S(y)| /|S(x)|=m\} ;
$$

choose $y \in L(x)$ at random (uniformly and independently for different $x$ ) and put $(x, y) \in \omega$. No other edges are put in $\omega$. Clearly every component of $\omega$ is infinite and the distribution of $\omega$ is $G$-invariant. To show that $\mathbf{E}[D(x)]=1+m$, we calculate that

$$
\mathbf{E}[D(x)]=\mathbf{E}\left[\sum_{y \in L(x)} \mathbf{1}_{\{(x, y) \in \omega\}}+\sum_{x \in L(z)} \mathbf{1}_{\{(z, x) \in \omega\}}\right]
$$




$$
\begin{aligned}
& =1+\sum_{z \in V} \mathbf{1}_{\{x \in L(z)\}} \mathbf{P}[(z, x) \in \omega] \\
& =1+\sum_{z \in V} \mathbf{1}_{\{z \in L(x)\}} \mathbf{P}[(x, z) \in \omega]|S(z)| /|S(x)| \\
& =1+m \mathbf{E}\left[\sum_{z \in L(x)} \mathbf{1}_{\{(x, z) \in \omega\}}\right]=1+m,
\end{aligned}
$$

where we have used the Mass-Transport Principle in deriving (6.2).

To show that this bound is sharp in case $G$ is unimodular, we borrow a result from [BLPS1]: the wired uniform spanning forest of $X$ is a $G$ invariant percolation with every component infinite and expected degree of every vertex equal to 2 .

Corollary 6.2 (Monotonicity of unimodularity). For $G$ a closed subgroup of $\operatorname{Aut}(X)$, let $m(G)$ be the minimum of $|S(x)| /|S(y)|$ for $x, y$ neighbors. If $G$ is transitive and $G \subseteq H$ with $H$ a closed subgroup of $\operatorname{Aut}(X)$, then $m(G) \leqslant m(H)$. In particular, if $G$ is unimodular, then so is $H$.

Proof. Since $H$ must also be transitive, there is an $H$-invariant percolation on $X$ with infinite components and expected degree $1+m(H)$. Since $H \subseteq G$, the percolation is also $G$-invariant, whence $1+m(H) \geqslant 1+m(G)$.

Remark 6.3 (A proof of Theorem 3.4). We can also use Theorem 6.1 to complete our proof of Theorem 3.4 that we began in Remark 3.11: Assume that $X$ is amenable and $G$ acts quasi-transitively. Let $o \in V$ and define the $G^{\prime}$-transitive amenable graph $X^{\prime}$ as in Lemma 3.10. Let $\mathbf{P}$ be a $G^{\prime}$-invariant percolation on $X^{\prime}$ concentrated on configurations all of whose components are infinite. Let $K_{n}$ be Følner sets in $X^{\prime}$, i.e., $\left|\partial_{V} K_{n}\right| /\left|K_{n}\right| \rightarrow 0$. Fix $n$ and write $\omega \cap K_{n}$ as the disjoint union of $L_{n}$ connected graphs $\omega_{i}=\left(V_{i}, E_{i}\right)$. Since each component of $\omega$ is infinite, each $\omega_{i}$ intersects the boundary of $K_{n}$, whence $L_{n} /\left|K_{n}\right| \rightarrow 0$ as $n \rightarrow \infty$. Therefore, we have

$$
\begin{aligned}
\frac{1}{\left|K_{n}\right|} \sum_{x \in K_{n}} \operatorname{deg}_{\omega} x & \geqslant \frac{1}{\left|K_{n}\right|} \sum_{i=1}^{L_{n}} \sum_{x \in K_{n}} \operatorname{deg}_{\omega_{i}} x \\
& \geqslant \frac{1}{\left|K_{n}\right|} \sum_{i=1}^{L_{n}}\left(2\left|V_{i}\right|-1\right)=\frac{2\left|K_{n}\right|-L_{n}}{\left|K_{n}\right|} .
\end{aligned}
$$

Taking expectation gives

$$
\mathbf{E}[D(o)]=\frac{1}{\left|K_{n}\right|} \sum_{x \in K_{n}} \mathbf{E}\left[\operatorname{deg}_{\omega} x\right] \geqslant \frac{2\left|K_{n}\right|-L_{n}}{\left|K_{n}\right|} \rightarrow 2
$$


as $n \rightarrow \infty$, whence by Theorem $6.1, G^{\prime}$ is unimodular. By Lemma 3.10, also $G$ is unimodular. It follows from Theorem 3.9 that $G^{\prime}$ is amenable and hence, by Lemma 3.10 again, that $G$ is amenable.

We now extend Theorem 6.1 to the quasi-transitive case:

Theorem 6.4. Let $X$ be a graph, $G \subseteq \operatorname{Aut}(X)$ be a closed quasi-transitive subgroup, $o_{1}, \ldots, o_{L}$ be a complete set of representatives in $V$ of the orbits of $G$, and write $\tau(x):=i$ if $x \in G o_{i}$. Let $\mathbf{P}$ be a $G$-invariant bond percolation on $X$ that has infinite components with positive probability. Let $\mu_{i}:=\left|S\left(o_{i}\right)\right|$,

$$
m_{i}:=\min \left\{\mu_{\tau(y)} /|S(y)|: y \sim o_{i}\right\},
$$

and $p_{\infty, i}$ be the probability that $o_{i}$ is in an infinite component. Then

$$
\sum_{i} \frac{\mathbf{E}\left[D\left(o_{i}\right) ;\left|K\left(o_{i}\right)\right|=\infty\right]}{\mu_{i} m_{i}} \geqslant \sum_{i} \frac{1+\left(m_{i} \wedge 1\right)}{\mu_{i} m_{i}} p_{\infty, i}
$$

Proof. The argument is very similar to the proof of Theorem 6.1. In fact, we use exactly the same mass transport. Define

$$
\Delta_{i}:=\sum_{z \in V} \theta\left(o_{i}, z\right)-\sum_{y \in V} \theta\left(y, o_{i}\right) \frac{|S(y)|}{\mu_{\tau(y)}} .
$$

Then Corollary 3.7 with $a_{i}:=1 / \mu_{i}$ implies that

$$
\sum_{i} \frac{\mathbf{E} \Delta_{i}}{\mu_{i}}=0
$$

Now $\Delta_{i}=0$ if $K\left(o_{i}\right)$ is finite. Otherwise, we again consider three cases: (1) If $D\left(o_{i}\right)=1$, then $\Delta_{i}=1$. (2) If $D\left(o_{i}\right) \geqslant 2$ and for some $z \neq o_{i}$, we have $\theta\left(o_{i}, z\right)=1$, then $\sum_{z \in V} \theta\left(o_{i}, z\right)=1$ and $\sum_{y \in V} \theta\left(y, o_{i}\right)|S(y)| / \mu_{\tau(y)} \leqslant$ $\left(D\left(o_{i}\right)-1\right) / m_{i}$, whence

$$
\Delta_{i} \geqslant 1-\left(D\left(o_{i}\right)-1\right) / m_{i} .
$$

(3) If $D\left(o_{i}\right) \geqslant 2$ and for all $z \neq o_{i}$, we have $\theta\left(o_{i}, z\right)=0$, then $\sum_{y \in V} \theta\left(y, o_{i}\right)|S(y)| / \mu_{\tau(y)} \leqslant\left(D\left(o_{i}\right)-2\right) / m_{i}$. Thus, in all three cases, we have

From (6.4), we obtain that

$$
\Delta_{i} \geqslant\left(1 \wedge \frac{1}{m_{i}}\right)-\frac{D\left(o_{i}\right)-1}{m_{i}}
$$

$$
\begin{aligned}
0 & =\sum_{i} \frac{\mathbf{E} \Delta_{i}}{\mu_{i}}=\sum_{i} \frac{\mathbf{E}\left[\Delta_{i} ;\left|K\left(o_{i}\right)\right|=\infty\right]}{\mu_{i}} \\
& \geqslant \sum_{i} \frac{1}{\mu_{i}} \mathbf{E}\left[\left(1 \wedge m_{i}^{-1}\right)-\left(D\left(o_{i}\right)-1\right) / m_{i} ;\left|K\left(o_{i}\right)\right|=\infty\right],
\end{aligned}
$$

which is the same as (6.3). 


\section{Ends of Components}

An end of a graph $X$ is an equivalence class of infinite nonself-intersecting paths in $X$, with two paths equivalent if for all finite $A \subset X$, the paths are eventually in the same connected component of $X-A$. An elementary application of the Mass-Transport Principle allows us to count the ends of the components in the configuration of a percolation that is invariant under a unimodular automorphism group:

Proposition 7.1 (One, two, or infinity ends). Let $G$ be a closed unimodular subgroup of $\operatorname{Aut}(X)$ that acts transitively on $X$. Let $\omega$ be the configuration of a $G$-invariant percolation on $X$ such that $\omega$ has infinite components with positive probability. Almost surely every component of $\omega$ with at least 3 ends has infinitely many ends.

Proof. Let $\omega_{1}$ be the union of the components $K$ of $\omega$ whose number $n$ of ends is finite and at least 3. Given a component $K$ of $\omega_{1}$, there is a connected subgraph $A \subset K$ with minimal $|V(A)|$ such that $K-A$ has $n$ infinite components. Let $H(K)$ be the union of all such subgraphs $A$. It is easy to verify that any two such subgraphs $A$ must intersect, and therefore $H(K)$ is finite. Let $H\left(\omega_{1}\right)$ be the union of all $H(K)$, where $K$ ranges over the components of $\omega_{1}$. Begin with unit mass at each vertex $x$ that belongs to a component $K$ of $\omega_{1}$, and transport it equally to the vertices in $H(K)$. Then the vertices in $H\left(\omega_{1}\right)$ receive infinite mass. By the Mass-Transport Principle, no vertex can receive infinite mass, which means that $\omega_{1}$ is empty a.s.

A forest is a graph all of whose components are trees. The following theorem was shown when $X$ is a tree and $G=\operatorname{Aut}(X)$ by Häggström $([\mathrm{H} 1])$.

Theorem 7.2 (Ends, $p_{c}$ and degrees). Let $G$ be a closed unimodular subgroup of $\operatorname{Aut}(X)$ that acts transitively on $X$. Let $\omega$ be the configuration of a $G$-invariant percolation on $X$ such that $\omega$ has infinite components with positive probability. If

(i) some component of $\omega$ has at least 3 ends with positive probability, then

(ii) some component of $\omega$ has $p_{c}<1$ with positive probability and

(iii) for every vertex $x, \mathbf{E}[D(x)|| K(x) \mid=\infty]>2$.

If $\omega$ is a forest a.s., then the three conditions are equivalent. 
REMARK 7.3. It follows from Theorem 7.2 that almost surely every component that has at least 3 ends has $p_{c}<1$. For suppose the contrary, and condition on having some component with at least 3 ends and $p_{c}=1$. Then the collection of all such components gives a $G$-invariant percolation that contradicts the theorem. Another conclusion is that if a.s. $\omega$ has a component with 3 ends, then a.s. it has a component with $p_{c}<1$.

Lemma 7.4 (Trimming to a forest). Let $G$ be a closed subgroup of Aut $(X)$. Let $\omega$ be the configuration of a $G$-invariant bond percolation on $X$. Then (on a larger probability space) there is a percolation with configuration $\omega^{\prime} \subset \omega$ such that the distribution of $\left(\omega^{\prime}, \omega\right)$ is $G$-invariant and that enjoys the following properties: For $x \in V$, let $K(x), K^{\prime}(x)$ denote the component of $x$ in $\omega, \omega^{\prime}$, resp. Then

(i) $\omega^{\prime}$ is a forest a.s.;

(ii) $\forall x \in V\left|K^{\prime}(x)\right|=|K(x)|$ a.s.; and

(iii) $\forall x \in V$ and $k \in \mathbb{Z}^{+}$, if $K(x)$ has at least $k$ ends with positive probability, then $K^{\prime}(x)$ also has at least $k$ ends with positive probability.

Furthermore, if $G$ is unimodular, then for all $x \in V$ such that $K(x)$ has at least 3 ends, there is a component of $K(x) \cap \omega^{\prime}$ that has infinitely many ends a.s.

Proof. Use the so-called free minimal spanning forest of $\omega$ : Assign to the edges i.i.d. uniform $[0,1]$ random variables. An edge $e \in \omega$ is present in $\omega^{\prime}$ iff there is no cycle in $\omega$ containing $e$ in which $e$ is assigned the maximum value. The first two properties are straightforward and well known. To see the third, assume that $K(x)$ has at least $k$ ends with positive probability. Choose any ball $A$ about $x$ so that $K(x)-E(A)$ has at least $k$ infinite components with positive probability. Then with positive probability, $K(x)-E(A)$ has at least $k$ infinite components, all edges in $A$ are assigned values less than $1 / 2$, and all edges in $\partial_{E} A$ are assigned values greater than $1 / 2$. On this event, $\omega^{\prime}$ contains a spanning tree of $A \cap \omega$ that is part of a tree in $\omega^{\prime}$ with at least $k$ ends.

In case $G$ is unimodular, let $r_{x}=r(x, \omega)$ be the least radius $r$ of a ball $B=B(y, r)$ in $X$ such that $K(x)-B$ has at least 3 infinite components, if such an $r$ exists. If not, set $r(x, \omega):=\infty$. Note that $r(x, \omega)<\infty$ iff $K(x)$ has at least 3 ends. As in the proof of Proposition 7.1, the MassTransport Principle shows that given that $K(x)$ has at least 3 ends, there are a.s. infinitely many $y \in K(x)$ at pairwise distance at least $2 r_{x}+2$ from each other such that $K(x)-B\left(y, r_{x}\right)$ has at least 3 infinite components. 
For such $y$, the events that all edges in $B\left(y, r_{x}\right)$ are assigned values less than $1 / 2$ and all edges in $\partial_{E} B\left(y, r_{x}\right)$ are assigned values greater than $1 / 2$ are independent and have probability bounded below, whence an infinite number of these events occur a.s. Therefore, there is a component of $K(x) \cap$ $\omega^{\prime}$ that has at least 3 ends a.s., whence, by Proposition 7.1, has infinitely many ends a.s.

Proof of Theorem 7.2. First consider the case where $\omega$ is a forest. The proof will be by throwing away a nonempty invariant collection of edges and appealing to Theorem 6.1.

Call a vertex $x$ an encounter point if its removal breaks $K(x)$ into at least 3 infinite components. For each encounter point $x$, choose one of its incident edges $e_{x}$ in $\omega$ at random (uniformly) and independently; let $\zeta$ be the set of edges chosen. Let $\eta_{1}$ be the set of edges $e_{x} \in \zeta$ such that there is a one-sided infinite path on $\omega$ starting at $x$ that passes through $e_{x}$ and does not contain any encounter point other than $x$. For each pair of encounter points $x \neq y$ such that the shortest path $\mathcal{P} \subset \omega$ from $x$ to $y$ does not go through any other encounter point and contains both $e_{x}$ and $e_{y}$, choose $e_{x}$ or $e_{y}$ at random with equal probability (and independently of other such decisions); let $\eta_{2} \subseteq \zeta$ be the resulting set of chosen edges. (Note that in case $x$ and $y$ are neighbors and $(x, y) \in \zeta$, then this means that $(x, y) \in \eta_{2}$.) Finally, set $\eta:=\eta_{1} \cup \eta_{2} \subseteq \zeta$.

A moment's thought shows that every vertex that is in an infinite component of $\omega$ is also in an infinite component of $\omega-\eta$.

For every vertex $x$, Theorem 6.1 implies that $\mathbf{E}\left[\operatorname{deg}_{\omega-\eta} x|| K(x) \mid=\infty\right]$ $\geqslant 2$. If (i) holds, then $\eta$ is nonempty with positive probability. Since $G$ acts transitively, this means that some edge incident to $x$ lies in $\eta$ with positive probability. Since $\eta \subseteq \omega$ contains only edges in infinite components of $\omega$, it follows that $\mathbf{E}[D(x)|| K(x) \mid=\infty]=\mathbf{E}\left[\operatorname{deg}_{\omega} x|| K(x) \mid=\infty\right]>2$, i.e., that (iii) holds.

Next, still assuming that $\omega$ is a forest, we deduce (ii) from (iii) by using Remark 4.3. Finally, that (ii) implies (i) for forests is trivial.

In case $\omega$ is not a forest a.s, choose $\omega^{\prime} \subset \omega$ as in Lemma 7.4. If (i) holds for $\omega$, then it also holds for $\omega^{\prime}$, whence (ii) and (iii) hold for $\omega^{\prime}$, whence also for $\omega$.

Corollary 7.5 (Small trees and expected degree). Let $G$ be a closed unimodular subgroup of $\operatorname{Aut}(X)$ that acts transitively on $X$ and let $\omega$ be the configuration of a $G$-invariant percolation on $X$. Fix a vertex $o$. Let $F_{o}$ be the event that $K(o)$ is an infinite tree with finitely many ends, and 
let $F_{o}^{\prime}$ be the event that $K(o)$ is a finite tree.

(i) If $P\left[F_{o}\right]>0$, then $\mathbf{E}\left[D(o) \mid F_{o}\right]=2$.

(ii) If $P\left[F_{o}^{\prime}\right]>0$, then $\mathbf{E}\left[D(o) \mid F_{o}^{\prime}\right]<2$.

Proof. For the first part, assume that all components of $\omega$ are infinite trees with finitely many ends. There is no loss of generality, since we can remove from $\omega$ all components that are not of this type. Since the components have only finitely many ends, they have $p_{c}=1$. Thus, Theorem 7.2 gives $\mathbf{E}\left[D(o) \mid F_{o}\right] \leqslant 2$. On the other hand, Theorem 6.1 gives $\mathbf{E}\left[D(o) \mid F_{o}\right] \geqslant 2$.

The second part is similar to Remark 4.3. Compare with Remarks 2.8 and 2.7.

EXAMPLE 7.6. We describe a transitive nonunimodular graph with an invariant random forest where each component has 3 ends, but the expected degree is smaller than 2 . Let $\mathbb{Z}_{3}:=\mathbb{Z} / 3 \mathbb{Z}$ be the group of order 3 and let $T$ be the 3 -regular tree with a distinguished end, $\xi$. On $T$, let every vertex be connected to precisely one of its offspring (as measured from $\xi$ ), each with probability $1 / 2$. Then every component is a ray. Let $\omega_{1}$ be the preimage of this configuration under the projection $T \times \mathbb{Z}_{3} \rightarrow T$. For every vertex $x$ in $T$ that has distance 5 from the root of the ray containing $x$, add to $\omega_{1}$ two edges at random in the $\mathbb{Z}_{3}$ direction, in order to connect the 3 preimages of $x$ in $T \times \mathbb{Z}_{3}$. The resulting configuration is a stationary spanning forest with 3 ends per tree and expected degree $(1 / 2) 1+(1 / 2) 2+2^{-(5+1)}((2 / 3) 1+(1 / 3) 2)$.

Similarly, one can construct a stationary spanning forest where each tree has one end and the expected degree is greater than 2. To do this, for every vertex $x$ that is a root of the ray in $T$, add to $\omega_{1}$ two edges at random in the $\mathbb{Z}_{3}$ direction to connect the 3 preimages of $x$, and for every edge $e$ in a ray in $T$ that has distance 5 from the root of the ray, delete two of its preimages at random.

Proof of Theorem 1.3. Let $\omega$ be the configuration of critical Bernoulli percolation on $X$. It is an easy well-known consequence of ergodicity that the number of infinite components for Bernoulli percolation is equal a.s. to a constant, which can only be 0,1 or $\infty$ (see [BuKe1]). Corollary 5.5 rules out a unique infinite cluster at $p=p_{c}(X)$. If at $p_{c}$, there were more than one infinite cluster, then by opening the edges in a large ball, we see that there would be, with positive probability, a cluster with at least 3 ends. This means, by Theorem 7.2, that with positive probability, some infinite component $K$ of $\omega$ has $p_{c}(K)<1$. Consequently, we may subtract from $\omega$ the configuration $\gamma_{\epsilon}$ of an independent Bernoulli $(\epsilon)$ percolation with 
$\epsilon>0$, such that with positive probability $\omega-\gamma_{\epsilon}$ has infinite components. However, this is impossible, as $\omega-\gamma_{\epsilon}$ is the configuration of a Bernoulli percolation with survival parameter smaller than $p_{c}$.

REMARK 7.7. It is possible, with some work, to extend the above proof of Theorem 1.3 to the quasi-transitive case. In [BLPS2], we present another proof of Theorem 1.3 that is very easy to adapt to the quasi-transitive setting.

\section{Invariant Percolation in the Hyperbolic Plane}

Theorem 8.1 (The number of infinite components). Let $G$ be a closed group of isometries of the hyperbolic plane $\mathbb{H}^{2}$. Suppose that $\mathcal{T}$ is a tiling of $\mathbb{H}^{2}$ by finite-sided compact polygons, that the isometries in $G$ are automorphisms of $\mathcal{T}$, and that $G$ acts quasi-transitively on $V$, where $X=(V, E)$ denotes the 1-skeleton (graph) of $\mathcal{T}$. Let $\omega$ be the configuration of a $G$ invariant bond percolation on $X$. Then the number of infinite components of $\omega$ is a.s. 0,1 or $\infty$.

REMARK 8.2. It follows that the total number of infinite monochromatic clusters in a stationary 2-coloring of the vertices of $X$ is a.s. 0,1 or $\infty$. This was proved for Bernoulli percolation assuming that $X$ has sufficiently large genus by Lalley ([L]), and stated without proof for the continuous Bernoulli Voronoi model by Benjamini and Schramm ([BSch2]).

Remark 8.3. Burton and Keane ([BuKe2]) described examples of Aut $\left(\mathbb{Z}^{2}\right)$ invariant percolation on $\mathbb{Z}^{2}$ with any finite number of infinite components. For example, let $T$ be the uniform spanning tree in $\mathbb{Z}^{2}$ and let $T^{\dagger}$ be the union of edges in the planar dual of $\mathbb{Z}^{2}$ that do not intersect edges of $T$. Then $T \cup T^{\dagger}$ can be thought of as an invariant percolation on a refined square lattice; it has 2 infinite components. An example with 3 infinite components is obtained by considering $T \cup T^{\dagger} \cup \Upsilon$ (in a further refined lattice), where $\Upsilon$ is the set of points in the plane that have the same distance from $T$ as from $T^{\dagger}$.

Proof of Theorem 8.1. Suppose that with positive probability, the number of infinite components in $\omega$ is $k$, where $2 \leqslant k<\infty$, and condition on this event. If $\omega$ has any finite components, absorb each finite component to an infinite component as follows. For each finite component $A$ that is adjacent to an infinite component, choose an edge in $\partial_{E} A$ at random that connects $A$ to an infinite component. Make these choices uniformly and 
independently. If $\omega$ still has finite components, then repeat this procedure at most countably many times until all components of $\omega$ are infinite. Since the result is still the configuration of a $G$-invariant percolation, we may assume from the start that a.s. $\omega$ has only infinite components and that their number is $k$.

It follows from the assumptions that $G$ is nonamenable. See, for example, [Z, Propositions 4.1.8 and 4.1.11]. We now define a $G$-invariant mean on $X$ and appeal to Lemma 3.3 to obtain a contradiction. Consider the edges in $X$ that join different components of $\omega$. Their dual edges in the planar dual of $X$ form a forest, $\Gamma$. It is not hard to verify that each component in $\Gamma$ has no leaves, the number of components of $\Gamma$ is finite and $\geqslant 1$, and the number of ends in any component of $\Gamma$ is finite and $\geqslant 2$.

We claim that a.s., all of the trees in $\Gamma$ have at most 2 ends. For if not, $\Gamma$ would have finitely many vertices of degree at least 3 . Each of these vertices is a face of $X$; let $K$ be the set of vertices (in $X$ ) in these faces. Since $K$ is finite, by taking at random a vertex from $K$, we could choose in an invariant way a random vertex of $X$, which is impossible since $G$ has infinite orbits. It follows that each component of $\Gamma$ is isomorphic to $\mathbb{Z}$.

Now, given $f \in \ell^{\infty}(V)$, assign each vertex $v$ in $\Gamma$ the average $\bar{f}(v)$ of the values of $f$ on the face corresponding to $v$.

Fix an enumeration of $V$. For each component $\Gamma_{i}$ of $\Gamma$, let $v_{i}$ be the first vertex in $\Gamma_{i}$ according to the enumeration. Let $M_{n}(f)$ denote the average of $\bar{f}$ on the union of the $n$-balls in $\Gamma$ centered at $v_{1}, \ldots, v_{k}$.

Given a Banach limit LIM, define $M(f):=\operatorname{LIM}_{n \rightarrow \infty} \mathbf{E} M_{n}(f)$. Then $M(f)$ does not depend on the choice of enumeration and is an invariant mean on $X$. By Lemma 3.3, this contradicts the nonamenability of $G$, and completes the proof.

Acknowledgement. We are grateful to Vadim Kaimanovich for pointing out the importance of unimodularity for our investigations. We thank Olle Häggström for a careful reading and corrections to an earlier version of our manuscript. We thank Wolfgang Woess for supplying us with his proof of the Mass-Transport Principle. Thanks are also due to Gabor Elek for bringing a reference to our attention.

\section{References}

[A] S. Adams, Trees and amenable equivalence relations, Ergodic Theory Dynamical Systems 10 (1990), 1-14. 
[ALy] S. AdAms, R. Lyons, Amenability, Kazhdan's property and percolation for trees, groups and equivalence relations, Israel J. Math. 75 (1991), $341-370$

[Al] K.S. Alexander, Percolation and minimal spanning forests in infinite graphs, Ann. Probab. 23 (1995), 87-104.

[An] A. Ancona, Positive harmonic functions and hyperbolicity, in "Potential Theory, Surveys and Problems" (J. Král et al., eds.), Springer Lecture Notes in Math. 1344 (1988), 1-23.

[BLPS1] I. Benjamini, R. Lyons, Y. Peres, O. Schramm, Uniform spanning forests, preprint.

[BLPS2] I. Benjamini, R. Lyons, Y. Peres, O. Schramm, Critical percolation on any nonamenable group has no infinite clusters, Ann. Probab., to appear.

[BLySch] I. Benjamini, R. Lyons, O. Schramm, Percolation perturbations in potential theory and random walks, in "Random Walks and Discrete Potential Theory" (M. Picardello, W. Woess, eds.), Cambridge U. Press, Cambridge, 1999, to appear.

[BSch1] I. Benjamini, O. Schramm, Percolation beyond $\mathbb{Z}^{d}$, many questions and a few answers, Electronic Commun. Probab. 1, Paper no. 8 (1996), $71-82$.

[BSch2] I. Benjamini, O. Schramm, Percolation in the hyperbolic plane, extended abstract (1996).

[BSch3] I. Benjamini, O. Schramm, Every graph with a positive Cheeger constant contains a tree with a positive Cheeger constant, GAFA 7 (1997), 403-419.

[BSch4] I. Benjamini, O. Schramm, Percolation in the hyperbolic plane, in preparation.

[BeM] J. van Den Berg, R.W.J. Meester, Stability properties of a flow process in graphs, Random Structures Algorithms 2 (1991), 335-341.

[BiMoSh] N.L. Biggs, B. Mohar, J. Shawe-Taylor, The spectral radius of infinite graphs, Bull. London Math. Soc. 20 (1988), 116-120.

[BlW] J. Block, S. WeinBerger, Aperiodic tilings, positive scalar curvature and amenability of spaces, J. Amer. Math. Soc. 5 (1992), 907-918.

[BuKe1] R.M. Burton, M. Keane, Density and uniqueness in percolation, Commun. Math. Phys. 121 (1989), 501-505.

[BuKe2] R.M. Burton, M. Keane, Topological and metric properties of infinite clusters in stationary two-dimensional site percolation, Israel J. Math. 76 (1991), 299-316.

[C] J. Cheeger, A lower bound for the lowest eigenvalue of the Laplacian, Problems in Analysis (Sympos. Salomon Bochner, Princeton Univ., 1969), Princeton Univ. Press, Princeton, NJ (1970), 195-199.

[D] J. Dodziuk, Difference equations, isoperimetric inequality, and tran- 
sience of certain random walks, Trans. Amer. Math. Soc. 284 (1984), 787-794.

[DKen] J. Dodziuk, W.S. Kendall, Combinatorial Laplacians and isoperimetric inequality, in "From Local Times to Global Geometry, Control and Physics" (K.D. Elworthy, ed.), Wiley, New York, Pitman Research Notes Math. Ser. 150 (1986), 68-74.

[FFu] L.R. Ford Jr., D.R. Fulkerson, Flows in Networks, Princeton University Press, Princeton, NJ, 1962.

[GKeN] A. Gandolfi, M.S. Keane, C.M. Newman, Uniqueness of the infinite component in a random graph with applications to percolation and spin glasses, Probab. Theory Related Fields 92 (1992), 511-527.

[Ge] P. GERL, Random walks on graphs with a strong isoperimetric property, J. Theor. Prob. 1 (1988), 171-187.

[Gr] G.R. Grimmett, The stochastic random-cluster process and the uniqueness of random-cluster measures, Ann. Probab. 23 (1995), 1461-1510.

[GrN] G.R. Grimmett, C.M. Newman, Percolation in $\infty+1$ dimensions, in "Disorder in Physical Systems" (G.R. Grimmett, D.J.A. Welsh, eds.), Clarendon Press, Oxford (1990), 219-240.

[H1] O. HÄGGSTRÖM, Infinite clusters in dependent automorphism invariant percolation on trees, Ann. Probab. 25 (1997), 1423-1436.

[H2] O. Häggström, Uniform and minimal essential spanning forests on trees, Random Structures Algorithms 12 (1998), 27-50.

[HaSl] T. Hara, G. Slade, Mean-field behaviour and the lace expansion, in "Probability and Phase Transition (Cambridge, 1993)" (G.R. Grimmett, ed.) Kluwer Acad. Publ., Dordrecht (1994), 87-122.

[Har] T.E. HARRIS, A lower bound for the critical probability in a certain percolation process, Proc. Cambridge Philos. Soc. 56 (1960), 13-20.

[K] V.S. Kaimanovich, Dirichlet norms, capacities and generalized isoperimetric inequalities for Markov operators, Potential Anal. 1 (1992), 61-82.

[Kes1] H. Kesten, Symmetric random walks on groups, Trans. Amer. Math. Soc. 92 (1959), 336-354.

[Kes2] H. Kesten, Full Banach mean values on countable groups, Math. Scand. 7 (1959), 146-156.

[Kes3] H. Kesten, The critical probability of bond percolation on the square lattice equals 1/2, Comm. Math. Phys. 74 (1980), 41-59.

[L] S.P. Lalley, Percolation on Fuchsian groups, Ann. Inst. H. Poincaré Probab. Statist.34 (1998), 151-177.

[Ly] R. Lyons, Random walks and the growth of groups, C.R. Acad. Sci. Paris 320 (1995), 1361-1366.

[OWe] D.S. Ornstein, B. Weiss, Entropy and isomorphism theorems for actions of amenable groups, J. d'Analyse 48 (1987), 1-141.

[P] A.L.T. Paterson, Amenability, American Mathematical Soc., Provi- 
dence, 1988.

[Pe] R. Pemantle, Choosing a spanning tree for the integer lattice uniformly, Ann. Probab. 19 (1991), 1559-1574.

[PePer] R. Pemantle, Y. Peres, Nonamenable products are not treeable, preprint.

[SWo] L. Saloff-Coste, W. Woess, Computing norms of group-invariant transition operators, Combin. Probab. Comput. 5 (1996), 161-178.

[Sa] M. SalvatoRI, On the norms of group-invariant transition operators on graphs, J. Theor. Probab. 5 (1992), 563-576.

[Sc] G. Schlichting, Polynomidentitäten und Permutationsdarstellungen lokalkompakter Gruppen, Invent. Math. 55 (1979), 97-106.

[SoWo] P.M. SoArdi, W. Woess, Amenability, unimodularity, and the spectral radius of random walks on infinite graphs, Math. Z. 205 (1990), 471-486.

[TWer] B. Tóth, W. Werner, The true self-repelling motion, Proba. Theory Relat. Fields 111 (1998), 375-452.

[Tr] V.I. Trofimov, Groups of automorphisms of graphs as topological groups, Math. Notes 38 (1985), 717-720.

[V] N.Th. Varopoulos, Isoperimetric inequalities and Markov chains, J. Funct. Anal. 63 (1985), 215-239.

[Wo1] W. Woess, Topological groups and infinite graphs, Discrete Math. 95 (1991), 373-384.

[Wo2] W. WoEss, Topological groups and recurrence of quasi transitive graphs, Rend. Sem. Mat. Fis. Milano 1994, 64 (1996), 185-213.

[Wu C.C. Wu, Critical behavior of percolation and Markov fields on branching planes, J. Appl. Probab. 30 (1993), 538-547.

[Z] R.J. Zimmer, Ergodic Theory and Semisimple Groups, Birkhäuser, Boston, 1984.

Itai Benjamini \& Oded Schramm Russell Lyons $\quad$ Yuval Peres Math. Dept. Dept. of Math. Inst. of Math. Weizmann Inst. Sci. Indiana Univ. Hebrew Univ. Rehovot $76100 \quad$ Bloomington, IN 47405-5701 Givat Ram Israel USA $\quad$ Jerusalem 91904 Israel

E-mails: itai@wisdom.weizmann.ac.il schramm@wisdom.weizmann.ac.il rdlyons@indiana.edu peres@math.huji.ac.il

Submitted: April 1998 Revision: October 1998 